(c) 2001 International Press

Adv. Theor. Math. Phys. 5 (2001) 93-137

\title{
Standard Models from Heterotic
}

\section{M-theory}

\author{
Ron Donagi ${ }^{1}$, Burt A. Ovrut ${ }^{2}$, Tony Pantev ${ }^{1}$ and Daniel \\ Waldram $^{3} 4$ \\ ${ }^{1}$ Department of Mathematics, University of Pennsylvania \\ Philadelphia, PA 19104-6395, USA \\ ${ }^{2}$ Department of Physics, University of Pennsylvania \\ Philadelphia, PA 19104-6396, USA \\ ${ }^{3}$ Department of Physics, Joseph Henry Laboratories \\ Princeton University, Princeton, NJ 08540, USA \\ ${ }^{4}$ Theory Division, CERN CH-1211, Geveva 23, Switzerland
}

\begin{abstract}
We present a class of $N=1$ supersymmetric models of particle physics, derived directly from heterotic M-theory, that contain three families of chiral quarks and leptons coupled to the gauge group $S U(3)_{\mathrm{C}} \times S U(2)_{\mathrm{L}} \times U(1)_{\mathrm{Y}}$. These models are a fundamental form of "brane-world" theories, with an observable and hidden sector each confined, after compactification on a Calabi-Yau threefold, to a BPS threebrane separated by a five-dimensional bulk space with size of the order of the intermediate scale. The requirement of three families, coupled
\end{abstract}

e-print archive: http://xxx.lanl.gov/hep-th/9912208 
to the fundamental conditions of anomaly freedom and supersymmetry, constrains these models to contain additional fivebranes wrapped around holomorphic curves in the Calabi-Yau threefold. These fivebranes "live" in the bulk space and represent new, non-perturbative aspects of these particle physics vacua. We discuss, in detail, the relevant mathematical structure of a class of torus-fibered Calabi-Yau threefolds with non-trivial first homotopy groups and construct holomorphic vector bundles over such threefolds, which, by including Wilson lines, break the gauge symmetry to the standard model gauge group. Rules for constructing phenomenological particle physics models in this context are presented and we give a number of explicit examples.

\section{Introduction}

In fundamental work, it was shown by Hořava and Witten $[1,2]$ that if eleven-dimensional M-theory is compactified on the orbifold $S_{1} / Z_{2}$, a chiral $\mathcal{N}=1, E_{8}$ gauge supermultiplet must exist in the twisted sector of each of the two ten-dimensional orbifold fixed planes. They argued that this gave the low-energy description of the strongly coupled $E_{8} \times E_{8}$ heterotic string. This is relevant for phenomenological particle physics since, when compactified to four dimensions [3], such theories will exhibit the left-right asymmetry of quark and lepton electroweak couplings required to describe the weak interactions. It is important to note that, in this theory, the chiral gauge matter is confined solely to the orbifold planes, while pure supergravity inhabits the bulk space between these planes. Thus, Horava-Witten theory is a concrete and fundamental representation of the idea of a "brane-world".

Witten then showed [3] that, if further compactified to four dimensions on a Calabi-Yau threefold, the $\mathcal{N}=1$ supersymmetric low energy theory exhibits realistic gauge unification and gravitational coupling strength provided the Calabi-Yau radius, $R$, is of the order of inverse $10^{16} \mathrm{GeV}$ and that the orbifold radius, $\rho$, is larger than $R$. Estimates of $\rho / R$ vary from 4-5 to several orders of magnitude [3, 4]. Thus, Hořava-Witten theory has a "large" internal bulk dimension, although it is of order the intermediate scale and not the inverse $\mathrm{TeV}$-size, or larger, bulk dimensions, discussed recently $[5,6]$.

As in the case of the weakly coupled heterotic string, when compactifying the Hořava-Witten theory to lower dimensions, it is possible that all or, more typically, a subset of the $E_{8}$ gauge fields do not vanish classically in the internal Calabi-Yau threefold directions. Since these gauge fields "live" on the Calabi-Yau manifold, $3+1$-dimensional Lorentz invariance is left unbroken. Furthermore, demanding that the associated field strengths 
satisfy the "Hermitian Yang-Mills" constraints $F_{a b}=F_{\bar{a} \bar{b}}=g^{a \bar{b}} F_{a \bar{b}}=0$, ensures that $\mathcal{N}=1$ supersymmetry is preserved. These gauge field vacua have two important effects. First, they spontaneously break the $E_{8}$ gauge group. Suppose that the non-vanishing gauge fields are associated with the generators of a subgroup $G \subseteq E_{8}$. Then, at low energies, the $E_{8}$ gauge group is spontaneously broken to the commutant subgroup $H$, formed from those generators of $E_{8}$ which commute with $G$. This allows one, in principle, to reduce the $E_{8}$ gauge group to smaller and phenomenologically more interesting gauge groups such as unification groups $E_{6}, S O(10)$ and $S U(5)$ as well as the standard model gauge group $S U(3)_{\mathrm{C}} \times S U(2)_{\mathrm{L}} \times U(1)_{\mathrm{Y}}$. The second effect is on the spectrum of massless charged particles in the low-energy theory which arise from the dimensional reduction of fields associated with the broken generators. By choosing the gauge vacuum, one can control the number of families of chiral quarks and leptons on the orbifold fixed plane.

A new ingredient in compactifications of Horava-Witten theory is that there may also be M-theory fivebranes in the vacuum $[3,7]$. Requiring that supersymmetry and the 3+1-dimensional Lorentz invariance is unbroken restricts the form of the fivebranes. Their six-dimensional worldvolumes are required to span the external 3+1-dimensional space while wrapping around a real two-dimensional surface $W$ within the Calabi-Yau manifold. Thus each fivebrane is at a definite point in the $S^{1} / Z_{2}$ orbifold. Furthermore, in order to preserve $\mathcal{N}=1$ supersymmetry, the real surface $W$ in the Calabi-Yau threefold must be holomorphic [3], meaning it is defined by the vanishing of functions which depend only on the holomorphic and not the anti-holomorphic coordinates of the Calabi-Yau space. Since the surface has one complex dimension, we will often refer to it as a (complex) curve.

There is an important cohomological constraint which relates the number of fivebranes and the curves on which they are wrapped to properties of the gauge vacuum and the Calabi-Yau manifold [3]. Without fivebranes, this becomes a constraint directly relating the gauge vacuum to the CalabiYau space. One of the important properties of including fivebranes in the background is that it relaxes this condition, making it much easier to find gauge vacua with suitable unbroken gauge groups and low-energy particle content. As we will see, all the examples of realistic compactifications we will give necessarily include fivebranes.

We refer to the general compactification of Horava-Witten theory on backgrounds with arbitrary supersymmetric gauge fields and $M$ fivebranes as "heterotic M-theory". The simplest heterotic M-theory vacuum, with the spontaneous breaking of $E_{8}$ to $E_{6}$ by taking $G=S U(3)$ and identifying it with the spin connection of the Calabi-Yau threefold, the "standard embed- 
ding", was discussed in the original paper of Witten [3]. Such vacua do not contain fivebranes. A discussion of general gauge vacua in this context and their low-energy implications was presented in $[7,8]$, with some explicit orbifold examples given in [9]. Fivebranes were first introduced into heterotic M-theory vacua, and their properties discussed, in [7].

An important result of this analysis is that there is an effective fivedimensional theory $[10,11]$ which realizes the heterotic M-theory vacua as BPS "brane-worlds". The bulk theory is gauged supergravity with a negative cosmological constant, and the orbifold planes and fivebranes appear as an array of BPS three-branes domain walls. The branes coming from the orbifold planes can have negative tension and the full spacetime solution is very similar to the anti-de Sitter space solution of Randall and Sundrum [6]. In fact, if one chooses to fix the Calabi-Yau moduli, it precisely realizes the solution of $[6]$.

Having defined heterotic M-theory, the real question is whether explicit heterotic M-theory vacua exist with the required low-energy properties. As for weakly coupled heterotic string compactifications, the difficult part in finding such models is in constructing the gauge vacuum. Given a CalabiYau threefold $Z$, what supersymmetric non-Abelian gauge field vacuum configurations associated with a subgroup $G \subseteq E_{8}$ can be defined on it? Unfortunately, solving the six-dimensional Hermitian Yang-Mills constraints explicitly is rarely possible, even in flat space. One, therefore, must look for an alternative construction of these Yang-Mills connections. Such an alternative is to be found in the work of Donaldson [12] and Uhlenbeck and Yau [13], which recasts the problem in terms of holomorphic vector bundles. These authors proved that for each holomorphic vector bundle, with structure group $G$ over $Z$, satisfying the condition of being "semi-stable", there exists a solution to the six-dimensional Hermitian Yang-Mills equations, and conversely. Thus, the analytic problem of finding gauge vacua by solving the Hermitian Yang-Mills constraint equations over $Z$ is reduced to an essentially topological problem of constructing semi-stable holomorphic vector bundles over the same manifold.

It is not immediately clear that any simplification has been achieved, but indeed it has, since some methods for constructing semi-stable holomorphic vector bundles are known. In particular, bundles were constructed for Calabi-Yau manifolds which appear as complete intersections in weighted projective spaces $[14,15]$. Recently, however, there were important new results by several authors $[16,17,18]$ on the construction of semi-stable holomorphic vector bundles over elliptically fibered Calabi-Yau manifolds. These are manifolds with a fibered structure, where the fiber is a two-dimensional 
torus, and which admit a global section. They are of particular interest because the heterotic string compactified on such spaces has a dual F-theory description. One method, the spectral-cover construction, essentially uses T-duality on the elliptic fiber to form the bundle from a simpler T-dual configuration. The simplest examples are for structure groups $S U(n) \subset E_{8}$, but other structure subgroups are possible as well. Thus, using holomorphic vector bundles and the Donaldson-Uhlenbeck-Yau theorem, it has been possible to classify and give the properties of a large class of $S U(n)$ gauge vacua even though the associated solutions of the Yang-Mills equations are unknown. This one might call the "mathematicians" approach, but, at present, it seems to be by far the simplest solution to an important physical problem.

The new results on bundles on elliptically fibered Calabi-Yau threefolds allows one to construct a number of phenomenologically interesting gauge vacua. In $[19,20]$, using the construction of $[16,17,18]$ and results of $[21,22]$, three-family vacua with unification groups such as $E_{6}, S O(10)$ and $S U(5)$ were obtained, corresponding to vector bundle structure groups $S U(3), S U(4)$ and $S U(5)$ respectively. The classical moduli space of the fivebranes in such vacua was then discussed in [23]. However, it was not possible to break $E_{8}$ directly to the standard gauge group $S U(3)_{\mathrm{C}} \times S U(2)_{\mathrm{L}} \times U(1)_{\mathrm{Y}}$ in this manner.

A natural solution to this problem, and that utilized in this paper, is to use non-trivial Wilson lines to break the GUT group down to the standard gauge group [24, 25]. This requires that the fundamental group of the Calabi-Yau threefold be non-trivial. Unfortunately, one can show that all elliptically fibered Calabi-Yau threefolds are simply connected, with the exception of fibrations over an Enriques base. In this case, however, we demonstrated in [20] that the vacuum obtained via spectral covers is not consistent with the requirement of three families of quarks and leptons. Further progress clearly required resolution of this fundamental problem.

With this in mind, recall that an elliptic fibration is simply a torus fibration that admits a zero section. As we noted above, the requirement of a zero section severely restricts the fundamental group of the threefold to be, modulo the Enriques exception, trivial. However, if one lifts the zero-section requirement, and considers holomorphic vector bundles over torus-fibered Calabi-Yau threefolds without section, then one expects to find non-trivial first homotopy groups and Wilson lines in vacua that are consistent with the three-family requirement.

In this paper, we give the relevant mathematical properties of a specific class of torus-fibered Calabi-Yau threefolds without section and construct 
holomorphic vector bundles over such threefolds. The technique is familiar from standard heterotic string constructions [25]. One starts with an elliptically fibered Calabi-Yau threefold $X$ which has trivial fundamental group, but is chosen to have a discrete group of freely acting symmetries $\Gamma$. Modding out, by identifying points on $X$ related by $\Gamma$, one forms a new, smooth threefold $Z=X / \Gamma$ which has fundamental group $\pi_{1}(Z)=\Gamma$. However, in general, $Z$ no longer admits a global section, and so is torus-fibered but not elliptically fibered. To construct holomorphic vector bundles on the torus-fibered Calabi-Yau threefold $Z$, one finds those bundles on $X$ which are invariant under $\Gamma$. These then descend to bundles on $Z$.

We next use these results to explicitly construct a number of three-family vacua with unification group $S U(5)$ which is spontaneously broken to the standard gauge group

$$
S U(3)_{\mathrm{C}} \times S U(2)_{\mathrm{L}} \times U(1)_{\mathrm{Y}}
$$

by Wilson lines $[24,25]$. This is done by taking $\Gamma=\mathbb{Z}_{2}$. The restriction to this specific class of torus-fibered threefolds and the GUT group $S U(5)$ is for simplicity only. By the same techniques one can construct a much wider class of torus-fibrations with more general unification groups. This will be presented elsewhere. We also leave a detailed exploration of the phenomenological and cosmological aspects of these models to later publications.

In this paper, we explicitly do the following. Sections 2 and 3 give new mathematical results necessary for constructing semi-stable holomorphic bundles on a torus-fibered three-fold $Z$ which admits Wilson lines. We will not give all the derivations, but try and highlight the ideas and main results. More mathematical details will be given in [26]. In section 2, we discuss the general construction of $Z$, with $\pi_{1}(Z)=\mathbb{Z}_{2}$, as the quotient of elliptically fibered threefolds $X$ by an involution $\tau_{X}$. This leads one to consider threefolds $X$ which admit two sections. We present the general conditions for a freely acting involution $\tau_{X}$ on such an $X$, and give the general homology classes, effective curves and second Chern class of $X$. In section 3 , we construct semi-stable holomorphic vector bundles $V$ over $X$ from a spectral cover, describing how they arise essentially by the action of T-duality. This involves a number of new features, since the spectral cover in such manifolds has a subtle structure. We discuss the conditions for invariance of $V$ under the involution. This gives us a description of bundles over $Z$. The second and third Chern classes of these bundles are presented. As we note, this construction is fairly general. The essential point is to have an elliptically fibered threefold $X$ with an involution $\tau_{B}$ on the base $B$ with finitely many fixed points and a number of such cases can be constructed. 


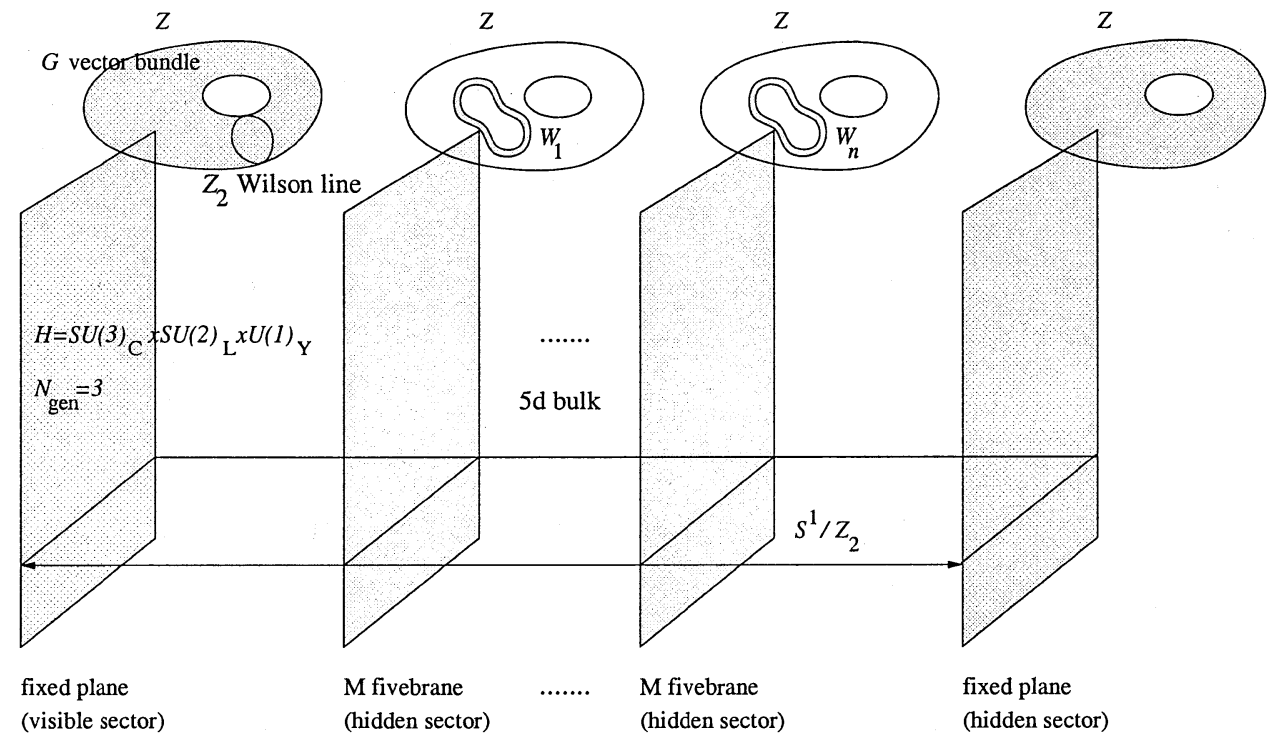

Figure 1: Heterotic M-theory brane-world

In the last sections we return to physics. In section 4 , we give explicit rules for the construction of three-family particle physics vacua on $Z$ with GUT group $S U(5)$. Since $\pi_{1}(Z)=\mathbb{Z}_{2}$, these vacua have Wilson lines that break $S U(5)$ to the standard $S U(3)_{\mathrm{C}} \times S U(2)_{\mathrm{L}} \times U(1)_{\mathrm{Y}}$ gauge group. In section 5 we present explicit examples of these "standard model" vacua for the base surfaces $B=F_{2}$ and $d P_{3}$ of the torus fibration. Finally, we summarize the resulting brane-world picture that emerges from these examples.

In conclusion, heterotic M-theory vacua give a concrete realization of the following "brane-world" (see Figure 1). Six of the eleven M-theory dimensions are compactified on a small Calabi-Yau manifold $Z$, typically on the scale of inverse $10^{16} \mathrm{GeV}$. The additional compact direction, the $S^{1} / Z_{2}$ orbifold, is larger, perhaps of order the intermediate scale. Matter is localized on the fixed planes of the $S^{1} / Z_{2}$ orbifold as well as on a number of fivebranes. (The exact matter content on the fivebranes depends on how they wrap the internal Calabi-Yau space.) In the effective five-dimensional theory, where the spacetime is the external $(3+1)$-dimensional space together with the $S^{1} / Z_{2}$ orbifold, the orbifold fixed planes and the fivebranes appear as a series of domain walls or BPS threebranes [10,11,7]. The fixed planes act as the boundaries of the orbifold interval, while the wrapped fivebranes appear as a series of threebranes arrayed throughout $S^{1} / Z_{2}$. By choosing an appropriate gauge vacuum, one can break one of the $E_{8}$ gauge groups on the orbifold fixed planes to the standard model group $S U(3)_{\mathrm{C}} \times S U(2)_{\mathrm{L}} \times U(1)_{\mathrm{Y}}$, 
with three families of chiral matter. Thus the standard model is realized on one fixed plane, while the other fixed planes and the fivebranes represent hidden sectors which only couple gravitationally to the standard model. Finally, we should note that related constructions, also using quotients of an elliptic Calabi-Yau threefold, were considered in [27]. However, we find that on a number of points our results are in disagreement with the calculations and conclusions of that paper.

\section{Fibered Calabi-Yau threefolds with non-trivial $\pi_{1}$}

In this section, we construct and describe the particular Calabi-Yau manifolds $Z$ we will use to compactify Hořava-Witten theory. Two properties are required. First, we must choose particular non-trivial gauge field configurations on the Calabi-Yau compactification to break the $E_{8}$ gauge group to a smaller GUT gauge group with three families. In this paper, we use the spectral-cover construction to describe these gauge field configurations. This construction requires the Calabi-Yau manifold to be elliptically fibered. The second condition comes from breaking the GUT group down to $S U(3)_{\mathrm{C}} \times S U(2)_{\mathrm{L}} \times U(1)_{\mathrm{Y}}$. This is most naturally accomplished by including non-trivial Wilson lines $[24,25]$ on the Calabi-Yau manifold $Z$. This requires that the threefold not be simply connected, that is, $\pi_{1}(Z)$ must be non-trivial.

It turns out that these two conditions are incompatible, except possibly in one case which is discussed in [20] and also below. Thus we are required to weaken one of the conditions and allow $Z$ to be fibered by tori which are not necessarily elliptic. We will review this distinction below.

To be specific, we will consider the case where the grand unified group is

$$
H=S U(5)
$$

It is well known $[24,25]$ that this can be broken to $S U(3)_{\mathrm{C}} \times S U(2)_{\mathrm{L}} \times U(1)_{\mathrm{Y}}$ by a $\mathbb{Z}_{2}$ Wilson line. Similar constructions work for other GUT groups, such as $S O(10)$ or $E_{6}$. Although our construction can be generalized to larger groups, in this paper we restrict our discussion to GUT group $S U(5)$ and to the simplest case of finding a torus-fibered Calabi-Yau threefold $Z$ with $\pi_{1}(Z)=\mathbb{Z}_{2}$. 


\subsection{Torus and elliptic fibrations}

By a torus-fibered threefold we mean a three-dimensional complex manifold $X$ which is equipped with a holomorphic map

$$
\pi: X \rightarrow B
$$

to some complex surface $B$ so that, for a general point $b \in B$, the fiber $\pi^{-1}(b)$ is a smooth complex curve of genus one. The surface $B$ is called the base of $X$. If one further demands that the canonical bundle be trivial, then $X$ is called a torus-fibered Calabi-Yau threefold. This condition is equivalent to assuming that

$$
c_{1}(T X)=0,
$$

where $c_{1}(T X)$ is the first Chern class of the tangent bundle $T X$. In this paper, $X$ will always be assumed to be a smooth manifold. Note, however, that the smoothness of $X$ does not imply the smoothness of the base $B$. In fact, bases with isolated singularities do occur in most of the interesting examples below.

Now consider a holomorphic map

$$
\sigma: B \rightarrow X,
$$

satisfying $\pi \circ \sigma=\mathrm{id}_{B}$. Such a map is called a section of $X$. It is important to note that a mapping of this type need not exist. Thus, there can be torus-fibered threefolds, including torus-fibered Calabi-Yau threefolds, that have no section. In fact, it is precisely such Calabi-Yau threefolds that will be required to construct realistic particle physics vacua.

Let us consider a torus-fibered threefold that does admit a section. Then the image, $\sigma(B)$, of the base in $X$ intersects each fiber $\pi^{-1}(b)$ at a unique point $\sigma(b)$. This point plays the role of a natural "zero" for the addition law on each fiber, turning each torus into an elliptic curve. For this reason, a torus fibration that admits a section is called an elliptic fibration. An elliptic fibration naturally possesses a line bundle, $\mathcal{L}$, on $B$ whose fiber at any point $b \in B$ is the cotangent line $T_{p}\left(\pi^{-1}(b)\right)$ to the elliptic curve at the zero point $p_{b}$. That is, $\mathcal{L}$ is the conormal bundle to the section $\sigma(B)$ in $X$. In the case where the elliptically fibered manifold is a Calabi-Yau threefold, the requirement that $c_{1}(T X)=0$ restricts the conormal bundle $\mathcal{L}$ to satisfy

$$
\mathcal{L}=K_{B}^{-1},
$$

where $K_{B}$ is the canonical bundle of the base $B$. This requirement restricts the possible bases $B$ [28]. It turns out that, for smooth $B$, the only 
possible cases are (i) the del Pezzo surfaces $d P_{i}$ for $i=1, \ldots, 9$, (ii) the Hirzebruch surfaces $F_{r}$ for any non-negative integer $r$, (iii) certain blow-ups of the Hirzebruch surfaces and (iv) the Enriques surface $\mathcal{E}$. Thus, elliptically fibered Calabi-Yau threefolds allow only a fairly restricted set of smooth base surfaces $B$. The properties of these bases are well known and reviewed in the Appendix of [20].

Elliptically fibered manifolds have a useful description in terms of a Weierstrass model. One recalls that a general elliptic curve can be embedded via a cubic equation into $\mathbb{C P}^{2}$. Without loss of generality, the equation can be put in the Weierstrass form

$$
z y^{2}=4 x^{3}-g_{2} x z^{2}-g_{3} z^{3} .
$$

where $g_{2}$ and $g_{3}$ are general coefficients and $(x, y, z)$ are homogeneous coordinates on $\mathbb{C P}^{2}$. To define an elliptic fibration over a base $B$, one needs to specify how the coefficients $g_{2}$ and $g_{3}$ vary as one moves around the base. In general, the coefficients must be sections of the line bundles $\mathcal{L}^{4}=K_{B}^{-4}$ and $\mathcal{L}^{6}=K_{B}^{-6}$ respectively. One notes that the torus fibers are actually elliptic curves, because there is always one solution to the Weierstrass equation, namely $(x, y, z)=(0,1,0)$. This defines a global section $\sigma$ of the fibration, usually called the "zero section".

The elliptic curve becomes singular when two roots of the Weierstrass equation (6) coincide. This occurs when the discriminant, defined by

$$
\Delta=g_{2}^{3}-27 g_{3}^{2}
$$

vanishes. In the fibration, the discriminant is a section of $\mathcal{L}^{12}=K_{B}^{-12}$. The set of points in the base over which the fibration becomes singular is given by the discriminant locus

$$
\Delta=0 .
$$

and defines a complex curve in the base.

In some cases, although some of the elliptic fibers are singular, the full space described by the Weierstrass model is smooth. However, as we will see below, the Weierstrass model may also be singular. In this case, the smooth Calabi-Yau threefold $X$ is a blow up of the corresponding singular Weierstrass model.

\subsection{Involutions and the construction of $Z$ with $\pi_{1}(Z)=\mathbb{Z}_{2}$}

Unfortunately, one can show that most elliptically fibered Calabi-Yau threefolds $Z$ have trivial $\pi_{1}(Z)$. The one exception is when the base $B$ is an 
Enriques surface. It was shown in [20] that spectral cover constructions on such geometries cannot give models with three families of chiral matter. Different constructions of bundles on such geometries do exist [29] but they do not seem to allow enough flexibility for satisfying the anomaly cancellation condition. Hence, we choose a different route; we keep the bases general but we relax the elliptic fibration condition.

The easiest way to produce a fibered space $Z$ with non-trivial $\pi_{1}(Z)$ is by quotienting an elliptically fibered space $X$ by a freely-acting discrete symmetry. Since here we consider only the simplest case of $\pi_{1}(Z)=\mathbb{Z}_{2}$, we need only find an elliptically fibered manifold $X$ with a freely-acting involution. Specifically, we would like to construct an involution

$$
\tau_{X}: X \rightarrow X
$$

that preserves the fibration $\pi$, as well as the holomorphic volume form, and acts freely on $X$, that is, without fixed points. We can then construct the quotient space

$$
Z=X / \tau_{X}
$$

Since $\tau_{X}$ acts freely and preserves the holomorphic volume form, $Z$ is a smooth Calabi-Yau threefold. However, in general, the section $\sigma$ of $X$ will not be invariant under $\tau_{X}$, so it will not descend to a section of $Z$. It follows that there is no reason to expect $Z$ to have a section and, hence, to be an elliptic fibration. It is, in general, only a torus-fibration.

How does one construct such an involution and what constraints does its existence place on $X$ ? This is most easily analyzed by constructing $\tau_{X}$ as the combination of two involutions, one acting on the base and one acting on the fiber. Note, first, that if there is an involution $\tau_{X}$ on $X$ that preserves the fibration, this must project to some involution

$$
\tau_{B}: B \rightarrow B
$$

on $B$. Since it is $X / \tau_{X}$ that must be smooth and not $B / \tau_{B}$, we do not require that $\tau_{B}$ act freely on $B$. In fact, generally $B$ will not admit a freely-acting $\tau_{B}$. We denote by $\mathcal{F}_{\tau_{B}}$ the set of points in $B$ that are fixed under $\tau_{B}$. In general, $\mathcal{F}_{\tau_{B}}$ can contain either a continuous or a finite set of elements.

Fix one such involution $\tau_{B}$. Among all Calabi-Yau manifolds $X$, fibered over $B$, we want to show there are some for which the involution $\tau_{B}$ lifts to an involution $\alpha: X \rightarrow X$ which preserves the section $\sigma$. We can then combine $\alpha$ with an involution $t_{\zeta}$ on the fibers, to give $\tau_{X}$. The lifting is possible only if the fibration is invariant under $\tau_{B}$. This means that the 
coefficients $g_{2}$ and $g_{3}$ in the Weierstrass equation must be invariant under $\tau_{B}$

$$
\tau_{B}^{*}\left(g_{2}\right)=g_{2}, \quad \tau_{B}^{*}\left(g_{3}\right)=g_{3},
$$

If these conditions are satisfied, then $\tau_{B}$ can indeed be lifted to an involution $\alpha: X \rightarrow X$, which is uniquely determined by the additional requirements that it fix the zero section $\sigma$ and that it preserve the holomorphic volume form on $X$. A local computation shows that this $\alpha$ leaves fixed the whole fiber above each fixed point in the base, that is all the points in $\mathcal{F}_{\tau_{B}}$. Thus it is not by itself a suitable candidate for $\tau_{X}$.

To construct an involution without fixed points, we combine $\alpha$ with a translation of the fiber. The action of translation on a smooth torus acts without fixed points. One might then expect that a simple translation on all the elliptic fibers of $X$ might by itself give an involution without fixed points. The problem is that the translation might not act freely on the singular fibers. Thus, in general, a translation alone is not a candidate for $\tau_{X}$ either. However, one recalls that $\alpha$ left invariant only those fibers above points in the base in $\mathcal{F}_{\tau_{B}}$. If we combine $\alpha$ with a translation in the fiber, then, provided none of the fibers above $\mathcal{F}_{\tau_{B}}$ are singular, the resulting transformation should act without fixed points.

Let us be more specific. The easiest (but not most general) way to construct an elliptically fibered Calabi-Yau threefold $X$ with a fiber translation symmetry is to require that $X$ has two sections. The zero section $\sigma$ marks the zero points $p_{b}$ on the fibers $\pi^{-1}(b)$. Suppose there is a second section $\zeta$. Consider any fiber $\pi^{-1}(b)$. Denote by $\zeta_{b}$ the unique point of intersection of this fiber with the image $\zeta(B)$ of the base in $X$. Let us further assume that, for any point $b \in B, \zeta_{b}$ is a point of order two in the fiber, that is, $\zeta_{b}+\zeta_{b}=\sigma(b)$. In terms of the sections themselves, this can be written as

$$
\zeta+\zeta=\sigma .
$$

Using this property, one can define an involution $t_{\zeta}: X \rightarrow X$ as follows. Let $x$ be any point in $X$. Then $x$ lies in a fiber $\pi^{-1}(b)$ for some $b \in B$. Define

$$
t_{\zeta}(x)=x+\zeta_{b}
$$

Clearly, $t_{\zeta}$ satisfies $t_{\zeta} \circ t_{\zeta}=\mathrm{id}_{X}$ and, hence, is an involution. Furthermore, $t_{\zeta}$ has the property that

$$
t_{\zeta}(\sigma)=\zeta, \quad t_{\zeta}(\zeta)=\sigma .
$$

exchanging the two sections. 
We then construct the mapping

$$
\tau_{X}: X \rightarrow X,
$$

defined by combining $\alpha$ with the fiber translation $t_{\zeta}$ as

$$
\tau_{X}=\alpha \circ t_{\zeta}
$$

Clearly $\tau_{X}$ is an involution on $X$. By construction, $\tau_{X}$ preserves the fibration $\pi$ and induces the involution $\tau_{B}$ on the base. Since $\alpha$ preserves the fiber, it leaves the two sections invariant

$$
\alpha(\sigma)=\sigma, \quad \alpha(\zeta)=\zeta
$$

Combined with the action of $t_{\zeta}$ given in (15), this implies that $\tau_{X}$ interchanges the two sections, so

$$
\tau_{X}^{*}(\sigma)=\zeta, \quad \tau_{X}^{*}(\zeta)=\sigma .
$$

Thus neither section is preserved under $\tau_{X}$ and, consequently, the quotient space $Z=X / \tau_{X}$ generically has no sections and so is only torus-fibered. Furthermore, since one can show that $t_{\zeta}$ preserves the holomorphic volume form, we can conclude that $\tau_{X}$ also preserves the holomorphic volume form.

Does $\tau_{X}$ have fixed points in $X$ ? In general, the answer is affirmative. However, as discussed above, if none of the fibers of the set of fixed points in the base $\mathcal{F}_{\tau_{B}}$ are singular then the action of $\tau_{X}$ is free. Thus $\tau_{X}$ will act freely on $X$ if and only if

$$
\mathcal{F}_{\tau_{B}} \cap\{\Delta=0\}=\emptyset,
$$

where $\{\Delta=0\}$ is the discriminant locus in the base $B$. Except for the case of the Enriques base, this then implies that $\mathcal{F}_{\tau_{B}}$ must consist of a finite number of fixed points.

Furthermore, since $\tau_{X}$ preserves the fibration $\pi$ of $X$ and induces the involution $\tau_{B}$ on $B$, it follows that $Z$ is a torus-fibration over the base space

$$
S=B / \tau_{B} .
$$

Recall that, since involution $\tau_{B}$ generically has fixed points, $S$, unlike $Z$, is generically not a smooth manifold.

In summary, we can construct a torus-fibered Calabi-Yau threefold with $\pi^{1}(Z)=\mathbb{Z}_{2}$ by the following quotient

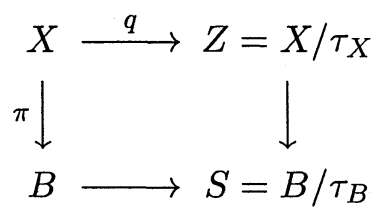

In order to construct such a manifold $Z$ we require 
- $X$ has two sections $\sigma$ and $\zeta$ such that $\sigma+\sigma=\zeta$ under fiber-wise addition,

- $\tau_{B}$ acts on the base $B$ with a fixed point set $\mathcal{F}_{\tau_{B}}$ with the property that $\mathcal{F}_{\tau_{B}} \cap\{\Delta=0\}=\emptyset$,

- in the Weierstrass model for $X$ we have $\tau_{B}^{*}\left(g_{2}\right)=g_{2}$ and $\tau_{B}^{*}\left(g_{3}\right)=g_{3}$, ensuring that the involution preserves the fibration.

It is worth noting that several explicit solutions to these conditions exist. In section 5 we give two examples based on $B=F_{2}$ and $B=d P_{3}$, but a number of other cases can also be found. In the next subsection, we will discuss what these conditions imply about the structure of $X$.

Finally, we note that the Chern classes $c_{i} \in H^{*}(Z, \mathbb{Q})$ of the tangent bundle $T Z$ can be determined from the Chern classes of $T X$ as follows. Let

$$
q: X \rightarrow Z
$$

be the quotient map. Since $X$ is a double cover of $Z$, it follows that

$$
c_{i}(T Z)=\frac{1}{2} q_{*} c_{i}(T X)
$$

where $q_{*} c_{i}(T X)$ is the push-forward of $c_{i}(T X)$.

\section{$2.3 \quad$ Structure of $X$}

The main requirement on $X$ is that it is a smooth elliptically fibered CalabiYau threefold admitting two sections. Such manifolds can be constructed from the corresponding Weierstrass model as follows. First, in order to have a pair of sections $\sigma$ and $\zeta$, the Weierstrass polynomial must factorize as

$$
z y^{2}=4(x-a z)\left(x^{2}+a x z+b z^{2}\right)
$$

where, comparing to equation (6), we see that

$$
g_{2}=4\left(a^{2}-b\right), \quad g_{3}=4 a b .
$$

Note that this implies that $a$ and $b$ are sections of $K_{B}^{-2}$ and $K_{B}^{-4}$ respectively. The zero section $\sigma$ is given by $(x, y, z)=(0,1,0)$ and the second section $\zeta$ by $(x, y, z)=(a, 0,1)$. The fibers are singular over the discriminant curve $\Delta=0$, where

$$
\Delta=\Delta_{1} \Delta_{2}^{2}
$$


and

$$
\Delta_{1}=a^{2}-4 b, \quad \Delta_{2}=4\left(2 a^{2}+b\right) .
$$

We see that the discriminant curve has two components.

As constructed, the Weierstrass model is singular. It is easy to show that there is a curve of singularities over the $\Delta_{2}=0$ component of the discriminant curve. We note that the vanishing of $\Delta_{2}$ corresponds to one of the roots of the second factor of the Weierstrass polynomial (25) being coincident with the zero of the $x-a z$ factor. Consequently, the singular points over the part of the discriminant curve where $\Delta_{2}=0$ all live in the $\zeta$ section. Specifically, the singular points form a curve $L$ in section $\zeta$ given by

$$
(x, y, z)=(a, 0,1), \quad 2 a^{2}+b=0
$$

To construct the smooth Calabi-Yau threefold, it is necessary to blow up this entire curve. (It turns out that one blowup suffices.) This is achieved as follows. The singular point of each individual fiber over $\Delta_{2}=0$ is replaced by a sphere $\mathbb{C P}^{1}$. This is a new curve in the Calabi-Yau threefold, which we denote by $N$. This reflects the fact that the general elliptic fiber $F$ has split over $\Delta_{2}=0$ into two spheres: the new fiber $N$ plus the proper transform of the singular fiber, which is in the class $F-N$. The union of these new fibers over the curve of singularities (29) forms a surface in the Calabi-Yau threefold, specifically, an exceptional divisor denoted by $E$. There is an analogous surface $E^{\prime}$ formed by the union of the $F-N$ fibers over $\Delta_{2}$ in the zero section. The blown-up Weierstrass model is the smooth elliptically fibered Calabi-Yau threefold $X$ with two sections. The blown up space together with the inverse image $\tilde{L}$ of the singular curve $L$ is shown in Figure 2.

We recall that to admit an involution we required in addition that $g_{2}$ and $g_{3}$ were invariant under $\tau_{B}$. In terms of the parameters $a$ and $b$ this translates into

$$
\tau_{B}^{*}(a)=a, \quad \tau_{B}^{*}(b)=b .
$$

The action of the involution $\tau_{X}$ can be seen in Figure 2. We recall that, under $\tau_{X}$, the two sections are exchanged. Similarly, the two surfaces $E$ and $E^{\prime}$ are exchanged. A generic fiber $F_{b}$ over a point $b \in B$, will be exchanged with the fiber $F_{b^{\prime}}$ over the image point $b^{\prime}=\tau_{B}(b)$ under the involution on $B$. 


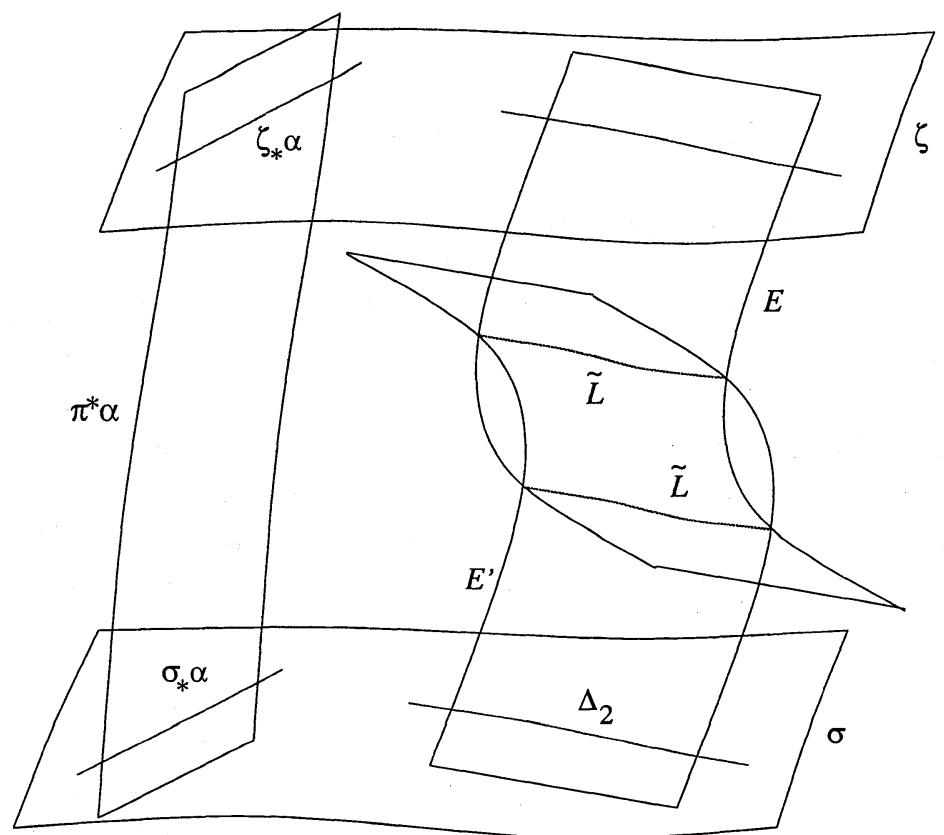

Figure 2: Structure of $X$

\subsection{Classes, effective curves and the Chern class $c_{2}(T X)$}

Let us consider the smooth elliptically fibered Calabi-Yau threefold $X$ with two sections, constructed as in the previous section. We would like to identify the independent classes of both surfaces and curves on $X$. For the classes of surfaces, generically, we have the classes of the two sections $\sigma$ and $\zeta$, of the new exceptional divisor $E$ obtained from the blowing-up procedure and the pull-back to $X$ of classes of curves $\alpha$ from the base $B$, which we denote by $\pi^{*} \alpha$. In fact, these divisor classes are not all independent. For the classes of curves, generically, we have the class of elliptic fiber $F$, of the new class $N$ obtained from the blowing-up procedure, the image $\tilde{L}$ of the curve of singularities and the embeddings via the two sections of classes of curves $\alpha$ from the base $B$, which we write as $\sigma_{*} \alpha$ and $\zeta_{*} \alpha$ respectively. Once again, these curve classes are not all independent. An independent set of divisors and curves, together with their intersections, is given by

\begin{tabular}{c|ccc} 
& $\pi^{*} \alpha$ & $\sigma$ & $\zeta$ \\
\hline$\sigma_{*} \beta$ & $\alpha \cdot \beta$ & $-c_{1} \cdot \beta$ & 0 \\
$F-N$ & 0 & 1 & 0 \\
$N$ & 0 & 0 & 1
\end{tabular}


where $\alpha$ and $\beta$ are classes of curves in $B$ and $c_{1}$ denotes the first Chern class of the base, $c_{1}(B)$.

The exceptional divisor from the blowing up procedure is given by

$$
E=2\left(\sigma-\zeta+\pi^{*} c_{1}\right)
$$

while it image $E^{\prime}$ under $\tau_{X}$ is

$$
E^{\prime}=2\left(\zeta-\sigma+\pi^{*} c_{1}\right) .
$$

Meanwhile, the transform of the curve of singularities is

$$
\tilde{L}=8\left[\sigma_{*} c_{1}+c_{1}^{2}(F-N)\right]
$$

and we have the important relationship between classes of curves

$$
\zeta \cdot \pi^{*} \alpha=\sigma \cdot \pi^{*} \alpha+\left(\alpha \cdot c_{1}\right)[(F-N)-N] .
$$

Finally, to complete the description of the cohomology ring we have the following intersections between divisors

$$
\begin{gathered}
\sigma \cdot \sigma=-\sigma_{*} c_{1}, \quad \zeta \cdot \zeta=-\zeta_{*} c_{1}, \\
\sigma \cdot \zeta=0, \\
\sigma \cdot \pi^{*} \alpha=\sigma_{*} \alpha, \quad \zeta \cdot \pi^{*} \alpha=\zeta_{*} \alpha
\end{gathered}
$$

The action of the involution $\tau_{X}$ on each class is easy to identify. We have for the divisors

$$
\begin{gathered}
\tau_{X}^{*}(\sigma)=\zeta, \quad \tau_{X}^{*}(\zeta)=\sigma \\
\tau_{X}^{*}\left(\pi^{*} \alpha\right)=\pi^{*} \tau_{B}(\alpha)
\end{gathered}
$$

while for the curves

$$
\begin{gathered}
\tau_{X}^{*}(F-N)=N, \quad \tau_{X}^{*}(N)=F-N, \\
\tau_{X}^{*} \sigma_{*} \alpha=\zeta_{*} \tau_{B}(\alpha)=\sigma_{*} \tau_{B}(\alpha)+\left(\alpha \cdot c_{1}\right)[(F-N)-N], \\
\tau_{X}^{*} \zeta_{*} \alpha=\sigma_{*} \tau_{B}(\alpha)
\end{gathered}
$$

When describing the fivebranes in our model, it is important to identify the effective classes of curves in $X$. As discussed in $[19,20,23]$, these are the cohomology classes which can be realized by the sum curves in the Calabi-Yau corresponding to an actual collection of fivebranes (and no antifivebranes). Not all classes are of this form. Thus we would like to identify the conditions for a class $[W]$ in $X$ to be effective, that is, for $[W]$ to lie in 
the Mori cone of $H_{2}(X, \mathbb{Z})$. We recall that in blowing up the Weierstrass model, the singular point of a fiber over the $\Delta_{2}=0$ was replaced by a sphere $\mathbb{C P}^{1}$ represented by the new class $N$. This class is by definition effective. Recall further that the fiber over $\Delta_{2}=0$ in fact splits into a pair of spheres. The other sphere is the proper transform of the singular fiber and is in the class $F-N$. It is by definition effective. Furthermore, the lift $\sigma_{*} \alpha$ of any effective class $\alpha$ in the base into the zero section $\sigma$ is must also be effective in $X$. It follows from (31) that we can, in general, write the class of a curve $W$ in $H_{2}(X, \mathbb{Z})$ as

$$
[W]=\sigma_{*} \omega+c(F-N)+d N,
$$

where $\omega$ is a class in the base $B$ and $c$ and $d$ are integers. It is easy to show that a sufficient condition for $W$ to be an effective class is that

$\omega$ is effective in $B, \quad c \geq 0, \quad d \geq 0 . \quad \Longrightarrow \quad[W]$ is effective in $\mathrm{X}$

We will, in the following, often denote an expression such as " $[W]$ is effective in $X$ " simply by $[W] \geq 0$.

It will be essential in this paper to know the second Chern class of the tangent bundle $T X$ of the relevant Calabi-Yau threefolds. The second Chern class $c_{2}(T X)$ of an elliptically fibered Calabi-Yau threefold with two sections can be calculated by blowing up the associated Weierstrass model, as discussed above. Here, we simply present the result. We find that

$$
c_{2}(T X)=12 \sigma \cdot \pi^{*} c_{1}+\left(c_{2}+11 c_{1}^{2}\right)(F-N)+\left(c_{2}-c_{1}^{2}\right) N,
$$

where $c_{1}$ and $c_{2}$ stand for the Chern classes $c_{1}(B)$ and $c_{2}(B)$ of the base $B$ respectively. This formula was originally given, though in a different form, by Andreas, Curio and Klemm [27]. Note that setting $N$ to zero reduces expression (41) to the original formula given by Friedman, Morgan and Witten [16] for the case of elliptically fibered Calabi-Yau threefolds with a single section $\sigma$.

\section{Holomorphic vector bundles on fibered Calabi- Yau threefolds}

Having identified the structure of the torus-fibered Calabi-Yau manifold $Z$ on which we will compactify, the next question is to construct general supersymmetric gauge vacua on $Z$, in particular, vacua with structure group $G=S U(5) \subset E_{8}$. As discussed in the introduction, constructing supersymmetric vacua corresponds to constructing general semi-stable holomorphic 
vector bundles over $\dot{Z}$. We recall that the work of $[16,17,18]$ shows how to construct such bundles over elliptically fibered manifolds. Here $Z$ is the quotient $X / \tau_{X}$ of an elliptically fibered threefold $X$ by an involution $\tau_{X}$. Thus the easiest way to construct bundles over $Z$ is to construct suitable vector bundles $V$ over $X$ which descend to the quotient threefold $Z$. This restricts us to those bundles on $X$ which are invariant under the involution. That is, we will need to find $V$ such that $\tau_{X}^{*}(V)=V$.

In this section, we discuss the details of such a construction. We first review the spectral cover construction of semi-stable holomorphic bundles $V$ over an elliptically fibered manifold $X$. We stress a physical picture, where the spectral data can be viewed as the T-dual of the gauge bundle $V$ over $X$. We then discuss the structure of the spectral data, in the case of the particular class of Calabi-Yau manifolds with two sections discussed in the previous section. Finally, we turn to the question of identifying those $V$ which are invariant under the involution. We also give explicit expressions for the Chern classes of $V$ and for the corresponding bundles on $Z$. A number of important subtleties complicate this analysis as compared with the generic case where $X$ has only one section. For simplicity, we will not give the derivations of all of our results in this paper. Also, we will often use the Chern classes to characterize a bundle rather than deal with the bundles themselves. The full mathematical details will be given elsewhere [26].

\subsection{T-duality and the spectral construction}

We would like to characterize semi-stable holomorphic $S U(n)$ vector bundles over an elliptically fibered Calabi-Yau threefold $X$. Let us generalize slightly and consider $U(n)$ bundles of degree zero over the fibers. It was shown in $[16,17,18]$ that, generically, a semi-stable rank $n$ holomorphic vector bundle $V$ on $X$ can be constructed from two objects

- a divisor $C$ of $X$ which is an $n$-fold cover of the base $B$, known as the spectral cover,

- a line bundle $\mathcal{N}$ on $C$.

The relationship between the spectral data $(C, \mathcal{N})$ and the bundle $V$ actually has a simple physical interpretation. The spectral data is the "T-dual" of the semi-stable holomorphic bundle $V$.

To understand the what is meant by T-duality in this context, recall that the Calabi-Yau manifold $X$ has an elliptically fibered structure. This means 
that we can consider making a T-duality transformation on the fiber. Specifically, on each fiber we make a T-duality transformation along each cycle of the torus, preserving the complex structure on the torus, but inverting the volume. Of course, the transformation is more subtle at the points where the fibration is singular, but it can in general be defined. The resulting Tdual manifold $\tilde{X}$, is also elliptically fibered. In fact, with regard to complex structure, it is isomorphic to the original manifold $X$. Mathematically, it is the "associated Jacobian bundle" $\widetilde{X} \equiv J(\pi)$. Thus we have

$$
X \stackrel{\text { T-duality }}{\longrightarrow} \tilde{X} \equiv J(\pi) \cong X
$$

by the action of T-duality on each fiber.

What happens to the vector bundle $V$ under such a duality transformation? Physically, we recall that vector bundles (or, more generally, sheaves) correspond to a collection of D-branes. The low-energy theory of $n$ coincident $\mathrm{D}$-branes is a $(D+1)$-dimensional Yang-Mills theory with a gauge group $U(n)$. From this point of view, we can view our rank $n$ vector bundle on the threefold $X$ as $n$ D6-branes all wrapping the Calabi-Yau manifold. If $V$ is non-trivial, the configuration is a source not only of D6-brane charge but also of D4-brane, D2-brane and D0-brane charge. The charges are given [30] in terms of the Chern character classes $\operatorname{ch}_{0}(V)=n, \operatorname{ch}_{1}(V), \operatorname{ch}_{2}(V)$ and $\operatorname{ch}_{3}(V)$ for D6-, D4-, D2- and D0-brane charge respectively. Thus, in general, the bundle describes some collection D6-, D4-, D2- and D0-branes.

This description can be extended to the case where we have, for instance, only D4-, D2- and D0-branes on $X$. This implies that the rank of the bundle is zero (no D6-brane charge) but the the higher Chern classes are non-zero. This is, of course, not possible to describe in terms of a vector bundle, but can be described as a "sheaf". A simple example is a single D4-brane wrapping a divisor $C$ in $X$. There will be a line-bundle $\mathcal{N}$ on $C$ describing the $U(1)$ gauge fields on $X$ (and in general describing embedded D2- and D0-brane charge). There is an inclusion map $i_{C}: C \rightarrow X$. We can try and use this map to push the bundle on $C$ into an object on $X$. This will be a "bundle" $i_{C *} \mathcal{N}$ which is simply $\mathcal{N}$ when restricted to the D4-brane on $C$ but everywhere else in $X$ the fiber is dimensionless. Such an object is a sheaf. A general vector bundle can then be regarded as a special type of sheaf.

Viewing $V$ as a set of $\mathrm{D}$-branes, we know that $\mathrm{D}$-branes are in general mapped under T-duality to new D-branes of different dimension. Roughly, D4-, D2- and D0-branes transverse to the fiber of $X$ should become D6-, D4- and D2-branes wrapping the fiber of $\widetilde{X}$, while D6-, D4- and D2-branes wrapping the fiber of $X$ become D4-, D2- and D0-branes transverse to the fiber of $\widetilde{X}$. (The actual transformation is more complicated because of the 
curvature of $X$ and the singular fibers, but this is the general picture.) Thus, in general, the bundle $V$ should map to some new bundle (or more generally new sheaf) $\widetilde{V}$ on $\widetilde{X}$ describing the T-dual configuration of D-branes. Since we can dualize back from $\widetilde{X}$ to $X$, the map is invertible. In what follows, it is more convenient to define the map in terms of the duality from the bundle $\widetilde{V}$ on $\widetilde{X}$ to $V$ on $X$. Thus, T-duality induces an invertible map $S$ between sheaves $\tilde{V}$ on $\widetilde{X}$ and sheaves $V$ on $X$ so that

$$
V=S(\widetilde{V}) \text {. }
$$

Mathematically, this map is known as the "Fourier-Mukai" transform. (To be more precise, this transform acts not on sheaves but on the derived category of sheaves on $X$ as described, for instance, in [31].) We note that it induces a map $s$ on cohomology exchanging the D-brane charges:

$$
\operatorname{ch}_{i}(V)=s \operatorname{ch}_{i}(\widetilde{V})
$$

We would expect that the T-duality acts linearly on the D-brane charges. And indeed, one can show that $s$ is linear on cohomology.

So far, while interesting, the T-duality map has not helped us solve the problem of characterizing semi-stable bundles $V$ on $X$. In general, the dual bundle $\widetilde{V}$ appears to be just as complicated as the original bundle $V$. However, we have not yet utilized the semi-stability condition. The spectral cover construction makes a specific semi-stability assumption, namely that the bundle is still semi-stable when restricted to a generic fiber ${ }^{1}$. The consequence of this is that if $V$ is semi-stable then its T-dual $\widetilde{V}=S^{-1}(V)$ describes only D4-branes and no D6-branes. In particular, this is precisely the information contained in the spectral data. Namely, the spectral cover $C$ is the surface in $\tilde{X}$ on which the D4-brane wraps and $\mathcal{N}$ describes the gauge field on the brane. Thus, formally, if

$$
i_{C}: C \rightarrow \tilde{X}
$$

is the inclusion map of the spectral cover, the T-dual $\widetilde{V}$ of $V$ is the sheaf

$$
\tilde{V}=i_{C *} \mathcal{N} .
$$

This then is the spectral cover construction

- the bundle $V$ is the fiber-wise T-dual or "Fourier-Mukai transform"

$$
V=S\left(i_{C *} \mathcal{N}\right)
$$

of the sheaf $\tilde{V}=i_{C *} \mathcal{N}$ as defined by the spectral data $(C, \mathcal{N})$.

\footnotetext{
${ }^{1}$ It is known [16] that for an appropriate choice of the Kähler form $\omega$ on $X$, o-stability indeed implies semi-stability on the generic fiber.
} 
We note that the spectral data naturally lives in the isomorphic T-dual space $\widetilde{X}$. The construction is shown diagrammatically in Figure 3 .
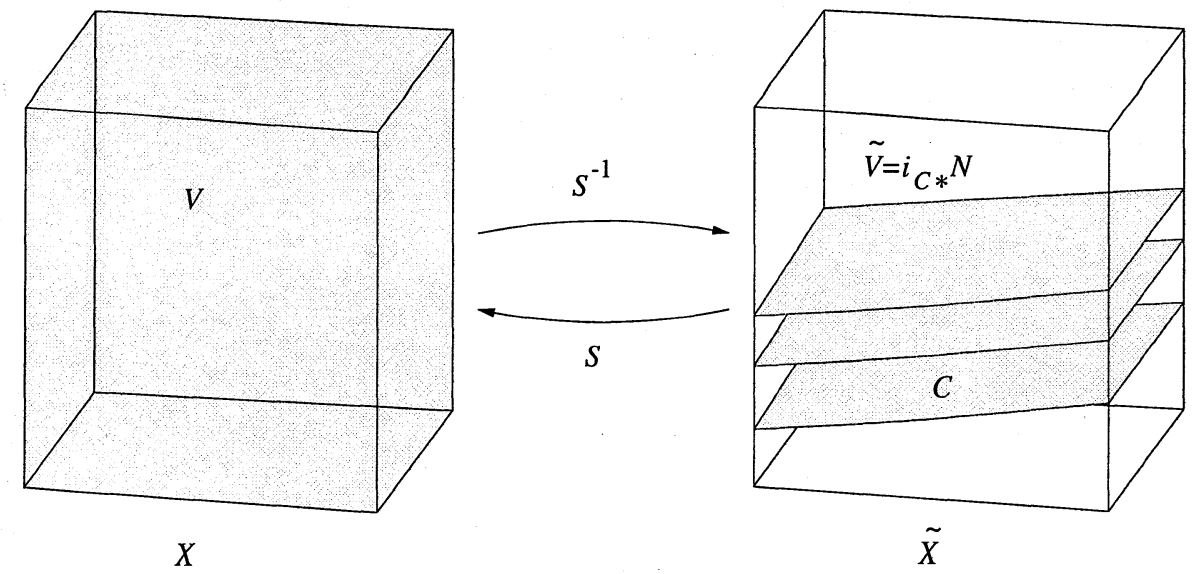

Figure 3: The Fourier-Mukai T-duality transform

One can get an idea of why the semi-stability condition implies that $\widetilde{V}$ contains no D6-branes as follows. It is easy to show that requiring the restriction of $V$ to a generic fiber to be semi-stable implies that first Chern class $c_{1}(V)$ restricted to a generic fiber is zero. This clearly restricts the form of the D4-brane charge. In particular, it means that there can be no D4-branes wrapping sections of $X$. However, it is precisely D4-branes wrapped on sections of $X$ which become D6-branes under T-duality since they are transverse to the fiber direction. Thus $\widetilde{V}$ contains only D4-, D2and D0-branes. Note that, in addition, the original $n$ D6-branes will map into $n$ D4-branes wrapping sections of the dual $\widetilde{X}$. To be more precise, they in general map to a D4-brane which is an $n$-fold cover of the base $B$. This is indeed precisely the form of the spectral cover $C$.

\subsection{Bundles on $X$ and the form of the spectral data}

Let us now return to the specific question of the description of semi-stable holomorphic $S U(n)$ bundles on $X$. We will find that the specific form we chose for $X$ gives additional degrees of freedom for the spectral data, which are not present in the generic case. In particular, as we discuss, the spectral cover is no longer everywhere a finite cover of the base $B$. In this paper, we will mention some of the complexities this introduces, but save the more mathematical analysis for a later publication [26]. 
First, we need to impose the condition that we have an $S U(n)$ bundle, so that

$$
c_{1}(V)=0,
$$

where $c_{1}(V)$ is the first Chern class of the bundle $V$. The map $s$ in cohomology induced by the Fourier-Mukai transform (44) gives expressions for the Chern classes $c_{i}(V)$ in terms of the Chern classes $c_{i}(\widetilde{V})$ of $\widetilde{V}=i_{C *} \mathcal{N}$. The latter are easily expressed in terms of $C$ and the first Chern class $c_{1}(\mathcal{N})$ of the line bundle $\mathcal{N}$. We will not give the details here but simply present the results. Requiring that $c_{1}(V)$ vanishes on $X$, rather than just on the fibers, imposes restrictions on $C$ and $\mathcal{N}$. We find that the class of the spectral cover is constrained to be

$$
C=n \sigma+\pi^{*} \eta,
$$

where $\pi^{*} \eta$ is the pull-back into $X$ of some divisor class $\eta$ in the base $B$. Since $C$ is an actual surface in $\widetilde{X}$, we have the addition condition that

$$
\eta \text { must be an effective class on } B
$$

Note, in addition, that equation (49) involves only the zero section $\sigma$ and not the second section $\zeta$. Furthermore, the vanishing of $c_{1}(V)$ also requires that

$$
c_{1}(\mathcal{N})=\left.\frac{1}{2}\left(C+\pi^{*} c_{1}\right)\right|_{C}+\gamma,
$$

where the first term is the restriction to $C$ of a divisor class in $X$, while $\gamma$ is a class on $C$ with the property that, if $p$ is the projection $p: C \rightarrow B$, then the push-forward of $\gamma$ vanishes

$$
p_{*} \gamma=0
$$

as an element of $H_{2}(B, \mathbb{Z})$.

We would like to have an explicit expression for the additional freedom $\gamma$ in the line bundle $\mathcal{N}$. To do this, we need to know the generic classes on the spectral cover $C$ and then find which of these classes satisfy the projection condition $p_{*} \gamma=0$.

If $C$ were completely generic, we would expect that the only classes on $C$ are those that come from the restriction of classes in $X$ to $C$. That is, we expect classes of the form $\gamma=\left.D\right|_{C}$ where $D \in H_{4}(X, \mathbb{Z})$ is a divisor class on $X$. From section 2.4, the divisors on $X$ are of the form $D=a \sigma+b \zeta+\pi^{*} \alpha$ where $\alpha$ is a class in $H_{2}(B, \mathbb{Z})$. However, we recall that the $c_{1}(V)=0$ 
condition meant that the spectral cover was not generic, but was restricted so that the class of $C$ is of the form $n \sigma+\pi^{*} \eta$. In this case, extra classes appear on the spectral cover. They can be seen by considering the intersection of $C$ with the exceptional divisor $E$. We have from (31) and (32) that, as a class in $X$,

$$
\left.E\right|_{C}=E \cdot C=2\left(\sigma-\zeta+\pi^{*} c_{1}\right) \cdot\left(n \sigma+\pi^{*} \eta\right)=4\left(\eta \cdot c_{1}\right) N
$$

Since both $C$ and $E$ are actual surfaces in $X$, this implies that they intersect in a curve wrapping the new fiber $4 \eta \cdot c_{1}$ times. For generic $C$, if we project down to the base, the curve $C \cdot E$ projects to $4 \eta \cdot c_{1}$ distinct points. Thus we can argue that, generically, the curve $C \cdot E$ splits into $4 \eta \cdot c_{1}$ distinct curves each wrapping the new component of the fiber over a different point in the base. In $X$, all of these curves are in the same homology class. However, in $C$, since each curve can be separately blown down, they must be in distinct cohomology classes in $H_{2}(C, \mathbb{Z})$. Let us denote these distinct classes as $N_{i}$ for $i=1, \ldots, 4 \eta \cdot c_{1}$. Thus, as a class in $C$, we have $\left.E\right|_{C}=\sum_{i} N_{i}$.

The fact that $C$ necessarily includes the exceptional curves $N_{i}$ has important consequences. In particular, it means that $C$ is no longer everywhere a finite cover of $B$. Generically it is an $n$-fold cover, but there are $4 \eta \cdot c_{1}$ special points in $B$ where it spans the whole of a new fiber $N$ above the base. This has important consequences for the spectral cover construction. In the previous section, we claimed that a smooth semi-stable holomorphic vector bundle $V$ on $X$ is the Fourier-Mukai transform of the sheaf $i_{C *} \mathcal{N}$ on $\widetilde{X}$, where $\mathcal{N}$ is a line-bundle on $C$. However, this is only strictly true for generic $C$. If $C$ is singular or is no longer a finite cover, more general sheaves on $\widetilde{X}$ can lead to a smooth semi-stable bundle $V$. In particular, one is led to consider sheaves supported on the new fibers $N_{i}$. We leave the discussion of these subtleties to a more mathematical paper [26]. In this paper, we will restrict ourselves to the simple case of line bundles over $C$, and will mostly been concerned with the Chern classes rather than the specific realization of the bundles themselves.

Returning to the classes on $C$, in summary, for a generic spectral cover $C=n \sigma+\pi^{*} \eta$ in $X$, the generic classes on $C$ can be taken as

$$
\begin{aligned}
\text { restrictions from } X: & \left.\sigma\right|_{C},\left.\quad \pi^{*} \alpha\right|_{C}, \\
\text { new fiber classes: } & N_{i},
\end{aligned}
$$

where $i=1, \ldots, 4 \eta \cdot c_{1}$. Furthermore, from their intersections in $X$ and the fact that each $N_{i}$ can be separately blown down, one can show that

$$
N_{i} \cdot N_{j}=-2 \delta_{i j}
$$


as an intersection in $C$, while

$$
\left.N_{i} \cdot \sigma\right|_{C}=\left.N_{i} \cdot \pi^{*} \alpha\right|_{C}=0
$$

for all $i$. One might expect that there is also a class from $\left.\zeta\right|_{C}$. However, we recall that $E=2\left(\sigma-\zeta+\pi^{*} c_{1}\right)$, so this class is not independent. We note, however, that $\frac{1}{2} E$ is also an integral class on $X$. Thus, we do have the useful fact that

$$
\left.\frac{1}{2} E\right|_{C}=\frac{1}{2} \sum_{i} N_{i}
$$

is actually an integral class on $C$, despite the rational coefficients in the sum.

Having identified the classes on $C$, we can now find the generic form of $\gamma$. A short calculation shows that $\gamma$ has the form

$$
\gamma=\lambda\left(n \sigma-\pi^{*} \eta+n \pi^{*} c_{1}\right) \cdot C+\sum_{i} \kappa_{i} N_{i} .
$$

In order for $c_{1}(\mathcal{N})$ to be an integral homology class, the coefficients $\lambda$ and $\kappa_{i}$ are restricted, so that, in the case where $n$ is odd, we have, recalling that $\frac{1}{2} \sum_{i} N_{i}$ is integral

$$
\begin{aligned}
\lambda-\frac{1}{2} & \in \mathbb{Z}, \\
\kappa_{i}-\frac{1}{2} m & \in \mathbb{Z},
\end{aligned}
$$

where $m$ is an integer. In this paper, we restrict our results to $n$ odd since all of the examples we present below will involve the structure group $S U(5)$.

The other Chern classes of $V$, which by the Fourier-Mukai transform could be written in terms of $C=n \sigma+\pi^{*} \eta$ and $\mathcal{N}$, then reduce to expression in terms of $\eta, \lambda$ and $\kappa_{i}$. We find that

$$
\begin{aligned}
c_{2}(V)= & \sigma \cdot \pi^{*} \eta-\left\{\frac{1}{24}\left(n^{3}-n\right) c_{1}^{2}-\frac{1}{2}\left(\lambda^{2}-\frac{1}{4}\right) n \eta\left(\eta-n c_{1}\right)-\sum_{i} \kappa_{i}^{2}\right\}(F-N) \\
& -\left\{\frac{1}{24}\left(n^{3}-n\right) c_{1}^{2}-\frac{1}{2}\left(\lambda^{2}-\frac{1}{4}\right) n \eta\left(\eta-n c_{1}\right)-\sum \kappa_{i}^{2}+\sum_{i} \kappa_{i}\right\} N,
\end{aligned}
$$

and

$$
c_{3}(V)=2 \lambda \eta\left(\eta-n c_{1}\right),
$$

where $c_{1}$ and $c_{2}$ stand for the Chern classes $c_{1}(B)$ and $c_{2}(B)$ of the base $B$ respectively. It is important to note that the expression for $c_{2}$ depends on 
the $\kappa_{i}$, but the one for $c_{3}$ does not. Setting $\kappa_{i}$ to zero in (60) returns one to the formula for $c_{2}$ given by Friedman, Morgan, Witten [16], as it must. The single section threefolds case of Expression (61) for $c_{3}(V)$ was derived in $[21]$.

\subsection{Vector Bundles on $Z=X / \tau_{X}$}

We now want to construct semi-stable holomorphic vector bundles $V_{Z}$ on the torus-fibration $Z=X / \tau_{X}$. Every such bundle can be pulled back to a bundle $V$ over $X$ that is invariant under the involution $\tau_{X}$ and conversely. That is, if

$$
\tau_{X}^{*}(V)=V
$$

then $V$ is the pull-back of a bundle $V_{Z}$ on $Z$. We therefore must characterize the condition that $V$ be invariant under $\tau_{X}$.

Recall that $V$ is constructed as the Fourier-Mukai transform of the sheaf $\widetilde{V}=i_{C *} \mathcal{N}$ given the spectral cover $C$ and the line bundle $\mathcal{N}$ on $C$. The generic forms of $C$ and $\mathcal{N}$ are given in equations (49) and (58) respectively. What additional conditions on the spectral data does the requirement (62) that $V$ is invariant under $\tau_{X}$ imply?

This can be calculated directly by finding the induced action of $\tau_{X}$ on the spectral data. In particular, if $S$ is the Fourier-Mukai transform from $\widetilde{V}$ to $V$, and $S^{-1}$ the inverse transform, we have that under $\tau_{X}$

$$
\widetilde{V}=i_{C *} \mathcal{N} \rightarrow \tilde{V}^{\prime}=S^{-1} \circ \tau_{X}^{*} \circ S\left(i_{C *} \mathcal{N}\right)
$$

Rather than considering this action in general, let us start by considering the condition invariance will place on the spectral cover, and then on the Chern classes of $\widetilde{V}$.

We recall that the spectral data live naturally in the T-dual space $\tilde{X}$. Furthermore, the involution $\tau_{X}$ is the combination of two involutions, $\tau_{X}=$ $\alpha \circ t_{\zeta}$, where $\alpha$ is the lift of the involution $\tau_{B}$ and $t_{\zeta}$ is a translation in the fiber of $X$. Under T-duality, a translation on the fiber of $X$ has no realization as an action on the dual space $\widetilde{X}$ so the induced action of $t_{\zeta}$ on $\widetilde{X}$ is trivial. The involution on the base $\tau_{B}$, however, carries over into the corresponding involution in the base of $\widetilde{X}=J(\pi)$. Thus, recalling that $X$ and $\widetilde{X}$ are isomorphic, we expect that one of the requirements of the invariance of $V$ under $\tau_{X}$ is that the T-dual D4-brane described by the spectral cover $C$ is invariant under $\alpha$. That is, we require that

$$
\alpha(C)=C
$$


Recall from (18) that $\sigma$ is invariant under $\alpha$. We find then, using the form of $C$ given in equations (49), that the condition (64) is satisfied provided

$$
\tau_{B}(\eta)=\eta
$$

It is more subtle, however, to calculate the induced transformation of $\mathcal{N}$ under the action of $\tau_{X}$ on $V$. Here, rather than calculate the action of $S^{-1} \circ \tau_{X}^{*} \circ S$ directly, we will consider the transformation of the Chern classes of $V$ to get information about how $\mathcal{N}$ transforms. Let us write $V^{\prime}=\tau_{X}^{*}(V)$ for the pullback of the bundle $V$ under the involution and $\eta^{\prime}, \lambda^{\prime}$ and $\kappa_{i}^{\prime}$ for the corresponding spectral data. Given the transformation of the classes on $X$ under $\tau_{X}$ given in (37) and (38), we find that, assuming the spectral cover condition (65) that $\eta^{\prime} \equiv \tau_{B}^{*}(\eta)=\eta$,

$$
\begin{aligned}
& c_{2}\left(V^{\prime}\right)=\tau_{X}^{*}\left(c_{2}(V)\right)=c_{2}(V)+\sum_{i}\left(\frac{1}{2}-\kappa_{i}\right)[N-(F-N)] \\
& c_{3}\left(V^{\prime}\right)=\tau_{X}^{*}\left(c_{3}(V)\right)=c_{3}(V)
\end{aligned}
$$

Comparing this to the general expressions (60) and (61) for $c_{2}(V)$ and $c_{3}(V)$, we can conclude that under $\tau_{X}$ we must have that $\lambda$ remains unchanged.

$$
\lambda \rightarrow \lambda^{\prime}=\lambda
$$

while for $\kappa_{i}$, we have

$$
\kappa_{i} \rightarrow \kappa_{i}^{\prime}
$$

such that

$$
\sum_{i} \kappa_{i}^{\prime}=\sum_{i}\left(\frac{1}{2}-\kappa_{i}\right)
$$

It might appear that there is also a condition on $\sum_{i} \kappa_{i}^{2}$. However, the additional fact that $S^{-1} \circ \tau_{X} \circ S$ is an involution, reduces this condition to that above.

In summary, necessary conditions for $V$ to be invariant are the relations

$$
\begin{aligned}
\tau_{B}(\eta) & =\eta, \\
\sum_{i} \kappa_{i} & =\sum_{i} \frac{1}{4}=\eta \cdot c_{1} .
\end{aligned}
$$

Note that, given the existence of a suitable three-fold $X$ with involution $\tau_{X}$, these conditions are completely general. Since the Chern classes do not completely determine the bundle, there may be non-invariant bundles 
with invariant Chern classes satisfying these conditions. To find the exactly invariant bundles requires the details of how the $\kappa_{i}$ transform individually.

Since physically we are interested mostly in the Chern classes, in this paper, for simplicity, we will restrict ourselves to the necessary conditions given above, without considering the details of identifying within this class of bundles the specific ones which are exactly invariant under $\tau_{X}$. Therefore, we take the condition that finding holomorphic vector bundles $V_{Z}$ over the torusfibered threefold $Z=X / \tau_{X}$ reduces to constructing holomorphic vector bundles $V$ over the elliptically fibered threefold $X$ that satisfy condition (70).

The Chern classes of a vector bundle $V_{Z}$ can be determined from the Chern classes of the $\tau_{X}$ invariant vector bundle $V$ from which it descends as follows. Let $q: X \rightarrow Z$ be the quotient map. Since $X$ is a double cover of $Z$, it follows that

$$
c_{i}\left(V_{Z}\right)=\frac{1}{2} q_{*} c_{i}(V),
$$

where $q_{*} c_{i}(V)$ is the push-forward of any Chern class $c_{i}(V)$ of $V$.

\section{Rules for Realistic Particle Physics Vacua}

In this section, we give the rules required to construct realistic particle physics vacua with $\mathcal{N}=1$ supersymmetry, three families of quarks and leptons with the standard model gauge group $S U(3)_{\mathrm{C}} \times S U(2)_{\mathrm{L}} \times U(1)_{\mathrm{Y}}$. For simplicity, we will restrict ourselves in this paper to considering vector bundles with the structure group $S U(n)$ for $n$ odd. In the observable sector we will choose the structure group $G=S U(5)$. The generalization to other structure groups is relatively straightforward.

The first set of rules deals with the selection of the elliptically fibered Calabi-Yau threefold $X$ which admits a freely-acting involution $\tau_{X}$, and the construction of bundles on $X$ invariant under $\tau_{X}$. As we have discussed, this allows us to construct a torus-fibered Calabi-Yau threefold $Z=X / \tau_{X}$, with non-trivial fundamental group $\pi_{1}(Z)=\mathbb{Z}_{2}$, and also to describe the bundles $V_{Z}$ on $Z$. These rules are summaries of the conditions we have already derived in the last two sections. If one was using this construction to build vector bundles for each of the two $E_{8}$ groups in Horava-Witten theory, then this first set of constraints is applicable to each bundle individually. The rules are 
- Involution conditions: Start with an elliptically fibered Calabi-Yau threefold $X$. If the base $B$ is smooth it must be either (i) a del Pezzo, (ii) a Hirzebruch, (iii) a blown-up Hirzebruch or (iv) an Enriques surface. To admit a freely-acting involution $\tau_{X}$, we required $X$ to have two sections $\sigma$ and $\zeta$. In the Weierstrass model, this corresponds to an explicit choice of

$$
g_{2}=4\left(a^{2}-b\right), \quad g_{3}=4 a b,
$$

with a discriminant curve

$$
\Delta=4\left(2 a^{2}+b\right)\left(a^{2}-4 b\right)=0,
$$

The existence of $\tau_{X}$ further required that

$$
\tau_{B}(a)=a, \quad \tau_{B}(b)=b
$$

where $\tau_{B}$ is the projection of $\tau_{X}$ onto the base, and in addition

$$
\mathcal{F}_{\tau_{B}} \cap\{\Delta=0\}=\emptyset,
$$

where $\mathcal{F}_{\tau_{B}}$ is the set of fixed points of $\tau_{B}$. It is helpful to note that this last condition implies that $\mathcal{F}_{\tau_{B}}$ must be finite, except when the base $B$ is a $d P_{9}$ or a $\mathrm{K} 3$ surface.

- Bundle condition: semi-stable holomorphic vector bundles over $X$ are given in terms of spectral data $(C, \mathcal{N})$. The condition that $c_{1}(V)=0$ implies that the spectral data can be written, via (49),(51),(58), in terms of a class $\eta$ in $B$ and coefficients $\lambda$ and $\kappa_{i}$, which satisfy

$$
\lambda-\frac{1}{2} \in \mathbb{Z}, \quad \kappa_{i}-\frac{1}{2} m \in \mathbb{Z},
$$

with $m$ integer. Furthermore, since $C$ is an actual surface in the Calabi-Yau manifold, we must also have

$$
\eta \text { is effective }
$$

as a class on $B$.

- Bundle involution conditions: in order for $V$ to descend to a vector bundle $V_{Z}$ over $Z$, the class $\eta$ in $B$ and the coefficients $\kappa_{i}$ must satisfy the constraints

$$
\begin{aligned}
\tau_{B}(\eta) & =\eta \\
\sum_{i} \kappa_{i} & =\eta \cdot c_{1} .
\end{aligned}
$$


The second set of rules is directly particle physics related. In general, in Hořava-Witten theory, we are free to choose two vector bundles, $V_{Z 1}$ and $V_{Z 2}$, located on the "observable" and "hidden" orbifold planes respectively. In this paper, for simplicity, we will always take $V_{Z 2}$ to be the trivial bundle. Hence, the gauge group $E_{8}$ remains unbroken on the hidden orbifold plane.

On the observable orbifold plane, the structure group of the bundle is $G \subset$ $E_{8}$. The commutant subgroup $H$ in $E_{8}$ is then the group preserved by the bundle. In this paper, we will spontaneously break $H$ to the standard model group $S U(3)_{\mathrm{C}} \times S U(2)_{\mathrm{L}} \times U(1)_{\mathrm{Y}}$ by means of a $\mathbb{Z}_{2}$ Wilson line on $Z[24,25]$. This is possible since, by construction, we have $\pi_{1}(Z)=\mathbb{Z}_{2}$. To achieve such a breaking requires us to take

$$
G=S U(5)
$$

so that the unification group will be

$$
H=S U(5) \text {. }
$$

Thus, we will assume that bundle on the observable plane $V_{Z 1}$ has structure group $G=S U(5)$.

The first of the particle physics conditions is the requirement that the theory have three families of quarks and leptons in the visible sector. The number of generations associated with the vector bundle $V_{Z 1}$ over $Z$ is given by [21]

$$
N_{\text {gen }}=\frac{1}{2} c_{3}\left(V_{Z 1}\right)
$$

Requiring $N_{\text {gen }}=3$ then, using (61) and (71), leads to the following rule for the associated vector bundle $V$ over $X$.

- Three-family condition: To have three families we must require

$$
\lambda \eta\left(\eta-n c_{1}\right)=6
$$

The second physical rule is associated with the anomaly cancellation requirement [3] that

$$
\left[W_{Z}\right]=c_{2}(T Z)-c_{2}\left(V_{Z 1}\right)-c_{2}\left(V_{Z 2}\right),
$$

where $\left[W_{Z}\right]$ is the class associated with non-perturbative five-branes in the bulk space of the theory. Since $V_{Z 2}$ is trivial by assumption, $c_{2}\left(V_{Z 2}\right)$ vanishes 
and condition (83) simplifies accordingly. Using equations (24), (71) and the fact that, by definition,

$$
\left[W_{Z}\right]=\frac{1}{2} q_{*}[W],
$$

condition (83) can be pulled-back onto $X$ to give

$$
[W]=c_{2}(T X)-c_{2}(V) .
$$

Inserting expressions (41) and (60) gives

$$
[W]=\sigma_{*} \omega+c(F-N)+d N
$$

where

$$
\omega=12 c_{1}-\eta
$$

and

$$
\begin{aligned}
& c=c_{2}+\left(\frac{1}{24}\left(n^{3}-n\right)+11\right) c_{1}^{2}-\frac{1}{2}\left(\lambda^{2}-\frac{1}{4}\right) n \eta\left(\eta-n c_{1}\right)-\sum_{i} \kappa_{i}^{2}, \\
& d=c_{2}+\left(\frac{1}{24}\left(n^{3}-n\right)-1\right) c_{1}^{2}-\frac{1}{2}\left(\lambda^{2}-\frac{1}{4}\right) n \eta\left(\eta-n c_{1}\right)-\sum_{i} \kappa_{i}^{2}+\sum_{i} \kappa_{i} .
\end{aligned}
$$

The class $\left[W_{Z}\right]$ must represent an actual physical holomorphic curve in the Calabi-Yau threefold $Z$ since physical five-branes are required to wrap around it. Hence, $\left[W_{Z}\right]$ must be an effective class, that is, $\left[W_{Z}\right]$ must be in the Mori cone of $H_{2}(Z, \mathbb{Z})$. It can be shown that $\left[W_{Z}\right]$ is effective in $Z$ if and only if its pull-back $[W]$ is an effective class in the covering threefold $X$. Therefore, we must require that $[W]$ be in the Mori cone of $H_{2}(X, \mathbb{Z})$. Using equation (40), this leads to the following rule.

- Effectiveness condition: For $[W]$ to be an effective class, we require

$$
\omega \text { is effective in } B, \quad c \geq 0, \quad d \geq 0 .
$$

Next, it is possible that our bundle with structure group $G$ may actually have a smaller structure group. If this is the case, then the preserved subgroup of $E_{8}$ will be larger than the commutant $H$ of $G$. Berglund and Mayer [32] have shown that, in the context of toric $X$, this will not be the case if the vector bundle satisfies a further constraint. (This was also discussed by Rajesh [33].) In the case of $S U(5)$ one requires 
- Stability condition: Let $G=S U(5) \subset E_{8}$ and $G$ be the structure group of the vector bundle. Then the commutant $H=S U(5)$ in $E_{8}$ will be the largest subgroup preserved by the bundle if

$$
\eta \geq 5 c_{1}
$$

Geometrically, this corresponds to requiring that the spectral cover does not split over the base $B$.

Finally, we recall that the point of constructing $Z$ with $\pi_{1}(Z)=\mathbb{Z}_{2}$ was that we can then include a $\mathbb{Z}_{2}$ Wilson line on $Z$ to break spontaneously the $H=S U(5)$ GUT group. We break

$$
S U(5) \rightarrow S U(3)_{\mathrm{C}} \times S U(2)_{\mathrm{L}} \times U(1)_{\mathrm{Y}},
$$

by assuming that the bundle contains a non-vanishing Wilson line with generator

$$
\mathcal{G}=\left(\begin{array}{ll}
\mathbf{1}_{3} & \\
& -\mathbf{1}_{2}
\end{array}\right)
$$

in $H=S U(5)$

If one follows the above rules, the vacuum will correspond to an $\mathcal{N}=1$ supersymmetric brane-world theory with, in the observable sector, three families of quarks and leptons and the standard model gauge group $S U(3)_{\mathrm{C}} \times S U(2)_{\mathrm{L}} \times U(1)_{\mathrm{Y}}$. Armed with these rules, we now turn to the explicit construction of phenomenologically relevant non-perturbative vacua.

\section{$5 \quad$ Three Family Models}

\subsection{Example 1: $B=F_{2}$}

In our first example, we take the base of the Calabi-Yau threefold to be the Hirzebruch surface

$$
B=F_{2} .
$$

As discussed in the Appendix of [20], the Hirzebruch surfaces are $\mathbb{C P}^{1}$ fibrations over $\mathbb{C P}^{1}$. There are two independent classes on $F_{2}$, the class of the base $\mathcal{S}$ and of the fiber $\mathcal{E}$. Their intersection numbers are

$$
\mathcal{S} \cdot \mathcal{S}=-2, \quad \mathcal{S} \cdot \mathcal{E}=1, \quad \mathcal{E} \cdot \mathcal{E}=0 .
$$


The first and second Chern classes of $F_{2}$ are given by

$$
c_{1}\left(F_{2}\right)=2 \mathcal{S}+4 \mathcal{E}
$$

and

$$
c_{2}\left(F_{2}\right)=4
$$

We now must show that there is an elliptically fibered Calabi-Yau threefold $X$ with $F_{2}$ base that admits a freely-acting involution $\tau_{X}$ satisfying the condition given in the previous section. The condition (75) implies that the projection $\tau_{B}$ of $\tau_{X}$ to the base has only a finite number of fixed points. To define $\tau_{B}$, we recall that there is a single type of involution on $\mathbb{C P}^{1}$. If $(u, v)$ are homogeneous coordinates on $\mathbb{C P}^{1}$, it can be written as $(u, v) \rightarrow(-u, v)$. This clearly has two fixed points, namely the origin $(0,1)$ and the point at infinity $(1,0)$ in the $u$-plane. To construct the involution $\tau_{B}$, we combine an involution on the base $\mathcal{S}=\mathbb{C P}^{1}$ with one on the fiber $\mathcal{E}=\mathbb{C P}^{1}$. We will not give details here, except to note that one finds that $\mathcal{F}_{\tau_{B}}$ contains four fixed points, coming from the fixed points of the $\mathbb{C P}^{1}$ fibers above the fixed points of the involution on the base. We should also point out that, in general, it is not possible to find involutions with a finite number of fixed points for all $F_{r}$.

To ensure that we can construct a freely-acting involution $\tau_{X}$ from $\tau_{B}$, we further need to show (75) that the discriminant curve can be chosen so as not to pass through these fixed points. We recall that the discriminant curve is given by

$$
4\left(2 a^{2}+b\right)^{2}\left(a^{2}-4 b\right)=0
$$

and that the parameters $a$ and $b$ are sections of $K_{B}^{-2}$ and $K_{B}^{-4}$ respectively, where $K_{B}$ is the canonical bundle of the base. In order to lift $\tau_{B}$ to an involution of $X$, we also require (74) that

$$
\tau_{B}(a)=a, \quad \tau_{B}(b)=b .
$$

This restricts the allowed sections $a$ and $b$ and, consequently, the form of $\Delta_{1}$ and $\Delta_{2}$. The question is then whether, within the class of allowed sections $a$ and $b$, there are examples where the corresponding discriminant curves avoid the fixed points. One can easily show that such sections $a$ and $b$ do exist.

To satisfy the conditions (78) on the invariance of $V$ under the involution, we need to find the classes $\eta$ in $F_{2}$ that are invariant under $\tau_{B}$. We find that the involution preserves both $\mathcal{S}$ and $\mathcal{E}$ separately, so that

$$
\tau_{B}(\mathcal{S})=\mathcal{S}, \quad \tau_{B}(\mathcal{E})=\mathcal{E} .
$$


Since any class $\eta$ is a linear combination of $\mathcal{S}$ and $\mathcal{E}$, we see that, in fact, an arbitrary $\eta$ satisfies $\tau_{B}(\eta)=\eta$.

We can now search for $\eta, \lambda$ and $\kappa_{i}$ satisfying the three family (82), effectiveness (90) and stability (91) conditions given above. We find that there are two classes of solutions

$$
\begin{array}{r}
\text { solution 1: } \quad \eta=14 \mathcal{S}+22 \mathcal{E}, \quad \lambda=\frac{3}{2}, \\
\sum_{i} \kappa_{i}=\eta \cdot c_{1}=44, \quad \sum_{i} \kappa_{i}^{2} \leq 60, \\
\text { solution 2: } \quad \eta=24 \mathcal{S}+30 \mathcal{E}, \quad \lambda=-\frac{1}{2}, \\
\sum_{i} \kappa_{i}=\eta \cdot c_{1}=60, \quad \sum_{i} \kappa_{i}^{2} \leq 76 .
\end{array}
$$

First note that the coefficients $\lambda$ satisfy the bundle constraint (76). Furthermore, one can find many examples of $\kappa_{i}$ with $i=1, \ldots, 4 \eta \cdot c_{1}$, satisfying the bundle constraint (76), the given conditions on $\sum_{i} \kappa_{i}^{2}$ and the invariance condition $\sum_{i} \kappa_{i}=\eta \cdot c_{1}$.

Using $n=5,(96),(101)$ and the intersection relations (95), one can easily verify that both solutions satisfy the three-family condition (82).

Next, from (86), (87), (88) and (89), as well as $n=5,(96),(97),(101)$ and the intersection relations (95), we can calculate the five-brane curves $W$ associated with each of the solutions. We find that

solution 1: $[W]=\sigma_{*}(10 \mathcal{S}+26 \mathcal{E})+(112-k)(F-N)+(60-k) N$,

solution 2: $[W]=\sigma_{*}(18 \mathcal{E})+(132-k)(F-N)+(76-k) N$,

where

$$
k=\sum_{i} \kappa_{i}^{2}
$$

It follows that the base components for $[W]$ are given by

$$
\begin{array}{ll}
\text { solution 1: } & \omega=10 \mathcal{S}+26 \mathcal{E}, \\
\text { solution 2: } & \omega=18 \mathcal{E}
\end{array}
$$

which are both effective. Furthermore, we note that for each five-brane curve the $c$ and $d$ coefficients of classes $F-N$ and $N$ respectively are non-negative integers (given the constraints (101) on $k$ ). Hence, effectiveness condition (90) is satisfied. 
Finally, note that the stability condition requires $\eta \geq 5 c_{1}$. In both of the above solutions

$$
\eta>10 \mathcal{S}+20 \mathcal{E}=5 c_{1},
$$

so that the condition is satisfied.

We conclude that, over a Hirzebruch base $B=F_{2}$, one can construct torus-fibered Calabi-Yau threefolds, $Z$, without section and with non-trivial first homotopy group $\pi_{1}(Z)=\mathbb{Z}_{2}$. Assuming a trivial gauge vacuum on the hidden brane, we have shown that we expect these threefolds to admit two classes of semi-stable holomorphic vector bundles $V_{Z},(101)$, associated with an $\mathcal{N}=1$ supersymmetric theory. These vacua have three families of chiral quarks and leptons and GUT group $H=S U(5)$ on the observable brane-world. Since $\pi_{1}(Z)=\mathbb{Z}_{2}$, Wilson lines break this GUT group

$$
S U(5) \rightarrow S U(3)_{C} \times S U(2)_{L} \times U(1)_{Y}
$$

to the standard model gauge group. Anomaly cancellation and supersymmetry require the existence of non-perturbative five-branes in the extra dimension of the bulk space. These five-branes are wrapped on holomorphic curves in $Z$ whose homology classes (102) are exactly calculable.

\subsection{Example 2: $B=d P_{3}$}

Let us now choose the base of the Calabi-Yau threefold $X$ to be

$$
B=d P_{3} .
$$

Recall that $d P_{3}$ can be thought of as the projective plane $\mathbb{C P}^{2}$ blown-up at three points. In terms of the homogeneous coordinates $(u, v, w)$ on $\mathbb{C P}^{2}$, the points we blow up may be taken to be

$$
(1,0,0), \quad(0,1,0), \quad(0,0,1) .
$$

The blownup surface $B$ can be embedded in $\mathbb{C P}^{6}$ with homogeneous coordinates $\left(z_{0}, \ldots, z_{6}\right)$ given by

$$
\left(z_{0}, \ldots, z_{6}\right)=\left(u v w, u^{2} v, w^{2} v, v^{2} w, u^{2} w, w^{2} u, v^{2} u\right)
$$

The blown-up points (108) then correspond to projective lines in $\mathbb{C P}^{6}$. For instance, considering the limit as $v$ and $w$ go to zero, we see that the point $(u, v, w)=(1,0,0)$ maps to the whole $\mathbb{C P}^{1}$ given by $\left(z_{0}, \ldots, z_{6}\right)=$ $(0, v, 0,0, w, 0,0)$ in $\mathbb{C P}^{6}$. 
The properties of del Pezzo surfaces were reviewed in the Appendix of [20]. For $d P_{3}$, one finds a basis for $H_{2}\left(d P_{3}, \mathbb{Z}\right)$ composed entirely of effective classes given by $l$ (the pullback of the hyperplane class of $\mathbb{C P}^{2}$ ) and three exceptional divisors $E_{i}$ for $i=1,2,3$ corresponding to the three blown-up points. Their intersection numbers are

$$
l \cdot l=1, \quad l \cdot E_{i}=0, \quad E_{i} \cdot E_{j}=-\delta_{i j} .
$$

In addition, there are three other dependent exceptional classes

$$
l-E_{2}-E_{3}, \quad l-E_{3}-E_{1}, \quad l-E_{1}-E_{2},
$$

corresponding to the lines in $\mathbb{C P}^{2}$ passing through pairs of the blown-up points. These lines are given by

$$
u=0, \quad v=0, \quad w=0
$$

respectively. The first and second Chern classes for $d P_{3}$ are

$$
c_{1}\left(d P_{3}\right)=3 l-E_{1}-E_{2}-E_{3},
$$

and

$$
c_{2}\left(d P_{3}\right)=6
$$

First, we must again show that the Calabi-Yau threefold $X$ based on $B=d P_{3}$ admits a freely-acting involution. The requirement (75) implies that the projection $\tau_{B}$ of $\tau_{X}$ to the base must have a finite number of fixed points. We start, therefore, by showing that such a $\tau_{B}$ exists. In terms of the $\mathbb{C P}^{2}$ coordinates $(u, v, w)$ define $\tau_{B}$ by

$$
\tau_{B}:(u, v, w) \rightarrow\left(u^{-1}, v^{-1}, w^{-1}\right) .
$$

In the ambiant space $\mathbb{C P}^{6}$ space this is simply the map exchanging $z_{1}$ and $z_{2}, z_{3}$ and $z_{4}$, and $z_{5}$ and $z_{6}$. Thus it is clearly an involution of the $d P_{3}$. Furthermore, it follows from (115) that $\tau_{B}$ has four isolated fixed points

$$
\mathcal{F}_{\tau_{B}}=\{(1, \pm 1, \pm 1)\}
$$

To ensure that we can construct a freely-acting involution $\tau_{X}$ from $\tau_{B}$, we need to show that the discriminant curve can be chosen so as not to intersect these fixed points. We recall that the two components of the discriminant curve are given by

$$
\Delta_{1}=a^{2}-4 b=0, \quad \Delta_{2}=4\left(2 a^{2}+b\right)=0,
$$


and that the parameters $a$ and $b$ are sections of $K_{B}^{-2}$ and $K_{B}^{-4}$ respectively, where $K_{B}$ is the canonical bundle of the base. The first Chern class of the anti-canonical bundle $K_{B}^{-1}$ is $c_{1}(B)$. Thus from (113), we see that as classes

$$
[a]=2\left(3 l-E_{1}-E_{2}-E_{3}\right), \quad[b]=4\left(3 l-E_{1}-E_{2}-E_{3}\right) .
$$

In terms of the blown-up $\mathbb{C P}^{2}, a$ and $b$ each correspond to curves. The function $a$ is a degree six homogeneous polynomial in $(u, v, w)$, describing a curve which passes twice through each of the blown-up points. The function $b$ is a degree twelve polynomial and describes a curve passing four times through each blow-up. These conditions restrict the form of the polynomials. For instance, all the terms involving fifth- or sixth-order powers of $u, v$ or $w$ are excluded in $a$. In addition, in order to lift $\tau_{B}$ to an involution of $X$, recall that we required

$$
\tau_{B}(a)=a, \quad \tau_{B}(b)=b .
$$

This means that the polynomials are further restricted to be homogeneous functions of $(u, v, w)$ which are invariant under the involution (115). It is a simple process to identify all such polynomials. The discriminant curves are then given by the vanishing of the twelfth-order polynomials $\Delta_{1}$ and $\Delta_{2}$. The question is then whether, within the class of allowed $a$ and $b$ polynomials, there are examples where the corresponding discriminant curves avoid the fixed points. In fact, from the form of $a$ and $b$, one can show that there is enough freedom in choosing the polynomials, so that any generic choice leads to discriminant curves which do not intersect any of the fixed points.

In order to have invariant bundles on $X$, by the condition (78), we need to find classes $\eta$ in $d P_{3}$ that satisfy $\tau_{B}(\eta)=\eta$. Using (108), (112) and the definition of $\tau_{B}$, we find that

$$
\begin{aligned}
& \tau_{B}\left(E_{1}\right)=l-E_{2}-E_{3}, \\
& \tau_{B}\left(E_{2}\right)=l-E_{3}-E_{1}, \\
& \tau_{B}\left(E_{3}\right)=l-E_{1}-E_{2} .
\end{aligned}
$$

Since the involution must preserve the intersection numbers and map effective curves to effective curves, this implies that

$$
\tau_{B}(l)=2 l-E_{1}-E_{2}-E_{3} .
$$

From these expressions, we see that there are three independent curves:

$$
\begin{aligned}
& a_{1}=l+E_{1}-E_{2}-E_{3}, \\
& a_{2}=l-E_{1}+E_{2}-E_{3}, \\
& a_{3}=l-E_{1}-E_{2}+E_{3},
\end{aligned}
$$


satisfying

$$
\tau_{B}\left(a_{i}\right)=a_{i}
$$

for $i=1,2,3$, and one perpendicular class

$$
p=l-E_{1}-E_{2}-E_{3},
$$

satisfying

$$
\tau_{B}(p)=-p .
$$

The three classes $a_{i}$ for $i=1,2,3$ generate all other $\tau_{B}$ invariant curves. Note that each class $a_{i}$ can be written as a sum of effective classes with nonnegative integer coefficients. For example, $a_{1}=E_{1}+\left(l-E_{2}-E_{3}\right)$. Hence, each $a_{i}$ is an effective class. It follows that the condition $\tau_{B}(\eta)=\eta$ can be solved simply by demanding that $\eta$ be written in terms of invariant classes only. That is

$$
\eta=m_{1} a_{1}+m_{2} a_{2}+m_{3} a_{3},
$$

where the $m_{i}$ are constant coefficients. In addition, note that the first Chern class of $d P_{3}$ given in (113) can be written as

$$
c_{1}=a_{1}+a_{2}+a_{3}
$$

and, hence, is invariant under $\tau_{B}$, as it must be.

We can now search for $\eta, \lambda$ and $\kappa_{i}$ satisfying the three family (82), effectiveness (90) and stability (91) conditions given above. We find that there are three classes of solutions

$$
\begin{gathered}
\text { solution 3: } \quad \eta=17 l-3 E_{1}-7 E_{2}-7 E_{3}, \quad \lambda=\frac{1}{2}, \\
\sum_{i} \kappa_{i}=\eta \cdot c_{1}=34, \quad \sum_{i} \kappa_{i}^{2} \leq 64, \\
\text { solution 4: } \quad \eta=18 l-2 E_{1}-8 E_{2}-8 E_{3}, \quad \lambda=\frac{1}{2}, \\
\sum_{i} \kappa_{i}=\eta \cdot c_{1}=36, \quad \sum_{i} \kappa_{i}^{2} \leq 66, \\
\text { solution 5: } \quad \eta=21 l+E_{1}-11 E_{2}-11 E_{3}, \quad \lambda=-\frac{1}{2}, \\
\sum_{i} \kappa_{i}=\eta \cdot c_{1}=42, \quad \sum_{i} \kappa_{i}^{2} \leq 72,
\end{gathered}
$$

First note that the coefficients $\lambda$ satisfy the bundle constraint (76). Furthermore, one can find many examples of $\kappa_{i}$ with $i=1, \ldots, 4 \eta \cdot c_{1}$, satisfying 
the bundle constraint (76), the given conditions on $\sum_{i} \kappa_{i}^{2}$ and the invariance condition $\sum_{i} \kappa_{i}=\eta \cdot c_{1}$.

Second, we see that each curve $\eta$ can be expressed as

$$
\begin{array}{ll}
\text { solution } 3: & \eta=7 a_{1}+5 a_{2}+5 a_{3} \\
\text { solution } 4: & \eta=8 a_{1}+5 a_{2}+5 a_{3} \\
\text { solution } 5: & \eta=11 a_{1}+5 a_{2}+5 a_{3}
\end{array}
$$

and, therefore, each $\eta$ is invariant under $\tau_{B}$. Using $n=5$, (113), (128) and the intersection relations (110), one can easily verify that all three solutions satisfy the three-family condition (82). Now, from (86), (87), (88) and (89), as well as $n=5,(113),(114),(128)$ and the intersection relations (110), we can calculate the five-brane curves $W$ associated with each of the three solutions. We find that

solution 3: $[W]=\sigma_{*}\left(5 a_{1}+7 a_{2}+7 a_{3}\right)+(102-k)(F-N)+(64-k) N$, solution 4: $\quad[W]=\sigma_{*}\left(4 a_{1}+7 a_{2}+7 a_{3}\right)+(102-k)(F-N)+(66-k) N$, solution 5: $[W]=\sigma_{*}\left(a_{1}+7 a_{2}+7 a_{3}\right)+(102-k)(F-N)+(72-k) N$,

where, as before,

$$
k=\sum_{i} \kappa_{i}^{2}
$$

It follows that the base components for $[W]$ are given by

$$
\begin{array}{ll}
\text { solution 3: } & \omega=5 a_{1}+7 a_{2}+7 a_{3}, \\
\text { solution 4: } & \omega=4 a_{1}+7 a_{2}+7 a_{3}, \\
\text { solution 5: } & \omega=a_{1}+7 a_{2}+7 a_{3} .
\end{array}
$$

Since the $a_{i}$ are effective, so is each of these $\omega$ classes. Furthermore, we note, given the conditions on $k$, that for each five-brane curve the $c$ and $d$ coefficients of the classes $F-N$ and $N$ respectively are all non-negative integers. Hence, the effectiveness condition (90) is satisfied.

Finally, note that the stability condition requires $\eta \geq 5 c_{1}$. In all of the above solutions

$$
\eta>5 a_{1}+5 a_{2}+5 a_{3}=c_{1}
$$

so the condition is satisfied.

We conclude that, over a del Pezzo base $B=d P_{3}$, one can also construct torus-fibered Calabi-Yau threefolds, $Z$, without section and with non-trivial 
first homotopy group $\pi_{1}(Z)=\mathbb{Z}_{2}$. Assuming a trivial gauge vacuum on the hidden brane, we have shown that we expect these threefolds to admit three families (39) of semi-stable holomorphic vector bundles $V_{Z}$, associated with an $\mathcal{N}=1$ supersymmetric theory with three families of chiral quarks and leptons and GUT group $H=S U(5)$ on the observable brane-world. Since $\pi_{1}(Z)=\mathbb{Z}_{2}$, Wilson lines break this GUT group

$$
S U(5) \rightarrow S U(3)_{C} \times S U(2)_{L} \times U(1)_{Y},
$$

to the standard model gauge group. Anomaly cancellation and supersymmetry require the existence of non-perturbative five-branes in the extra dimension of the bulk space. These five-branes are wrapped on holomorphic curves in $Z$ whose homology classes, (130), are exactly calculable.

\section{Conclusions}

In view of the technical nature of this paper, it is useful to conclude by focusing on the structure and physical properties of these "standard model" supersymmetric heterotic M-theory vacua.

The heterotic M-theory vacua discussed in this paper are constructed by compactifying Hořava-Witten theory on a smooth Calabi-Yau threefold, $Z$, one of a specific class with the property that

$$
\pi_{1}(Z)=\mathbb{Z}_{2}
$$

The threefolds discussed here were constructed from elliptically fibered CalabiYau threefolds, $X$, with two sections, by modding out a free involution that exchanges the two sections. The resulting Calabi-Yau spaces, $Z$, are torusfibered but not elliptically fibered since they admit no global sections. For instance in section 5, example 2 we constructed $Z$ from an elliptically fibered threefold $X$ with base $B=d P_{3}$, by modding out the involution which, restricted to the base, acts as

$$
(u, v, w) \rightarrow\left(u^{-1}, v^{-1}, w^{-1}\right),
$$

where $u, v$ and $w$ are complex coordinates on the $d P_{3}$.

The vacua of the effective low-energy theory consist of a large fivedimensional bulk space bounded on each end of the fifth dimension by a BPS domain wall with $\mathcal{N}=1$ supersymmetry on its world volume. A priori, these walls carry the $E_{8}$ gauge supermultiplets of the heterotic M-theory. 
We call one of the walls the "observable" sector and the other the "hidden" sector of the theory.

First consider the observable wall. In this paper, we constructed a class of smooth semi-stable holomorphic vector bundles over $X$ with the structure group

$$
G=S U(5)
$$

whose Chern classes are invariant under the involution $\tau_{X}$. The involution acts on this class of bundles; the invariant ones are precisely the bundles which come from $Z$. Their detailed construction is not required here since it is only the Chern classes that enter the physical discussion. The description of the invariant bundles will be given in a forthcoming paper [26].

Each such bundle corresponds to some $S U(5)$ instanton configuration on $Z$, which preserves $\mathcal{N}=1$ supersymmetry. However, it spontaneously breaks $E_{8}$ to the unification group $H=S U(5)$, which is the commutant of $G=S U(5)$ in $E_{8}$. Thus the gauge group on the observable wall is only $H=S U(5)$.

These bundles were constructed by first forming semi-stable holomorphic $S U(5)$ bundles over $X$ via the spectral cover construction, and then restricting to bundles that were invariant under the involution. Such bundles "descend" to $Z$. For instance, in example 3 in section 5 , the spectral data, specifying the bundle over $X$, were the class of a curve

$$
\eta=17 l-3 E_{1}-7 E_{2}-7 E_{3}
$$

in $B=d P_{3}$, together with the coefficients

$$
\lambda=\frac{1}{2}
$$

and $\kappa_{i}$ satisfying

$$
\sum_{i} \kappa_{i}=34, \quad \sum_{i} \kappa_{i}^{2} \leq 64 .
$$

This data specifies a class of $G=S U(5)$ semi-stable holomorphic bundle over $X$ whose Chern classes are invariant under the involution $\tau_{X}$. The involution $\tau_{X}$ therefore acts on the moduli space of these bundles. The fixed points of this action correspond to bundles which descend to a $G=S U(5)$ semi-stable holomorphic bundle on $Z$.

In addition, with the given choices of $\lambda$ and $\eta$, the associated vector bundle $V_{Z}$ over $Z$ has third Chern class $c_{3}\left(V_{Z}\right)=6$. This implies that the 
number of generations of quarks and leptons in the observable sector is

$$
N_{\text {gen }}=3 \text {. }
$$

Finally, since $\pi_{1}(Z)=\mathbb{Z}_{2}$, the $G=S U(5)$ gauge bundles can be "enlarged" by $\mathbb{Z}_{2}$ Wilson lines. These spontaneously break the unification group $S U(5)$ as

$$
S U(5) \rightarrow S U(3)_{C} \times S U(2)_{L} \times U(1)_{Y}
$$

on the observable domain wall.

Now consider the hidden wall. In this paper, for simplicity, we assume that we take a trivial vector bundle in this sector. Hence, $E_{8}$ is the unbroken gauge group on the hidden sector domain wall.

The vector bundles of the observable and hidden sectors are linked to the structure of $Z$ by the requirement of anomaly cancellation. Specifically, this relates the second Chern classes of the vector bundles to the second Chern class of the tangent bundle of $Z$. We find that, for three families in the observable sector, anomaly cancellation implies the existence of five-branes in the bulk space wrapped around a holomorphic curve in $Z$. The homology class of this curve, $W$, can be explicitly computed given $Z$ and the two vector bundles. For instance, for example 2 of section 5 , we showed that

$$
[W]=\sigma_{*}\left(19 l-9 E_{1}-5 E_{2}-5 E_{3}\right)+(102-k)(F-N)+(64-k) N
$$

where $k=\sum_{i} \kappa_{i}^{2}$. The generic structure of these "standard" model heterotic M-theory vacua is shown pictorially in Figure 1.

\section{Acknowledgments}

R. Donagi is supported in part by an NSF grant DMS-9802456 as well as a UPenn Research Foundation Grant. He was partially supported by a grant from the Emmy Noether Institute and the Minerva foundation of Germany, as well as by the Hebrew University of Jerusalem and RIMS, Kyoto during visits to those institutiuons. B. A. Ovrut is supported in part by a Senior Alexander von Humboldt Award, by the DOE under contract No. DE-AC02-76-ER-03071 and by a University of Pennsylvania Research Foundation Grant. T. Pantev is supported in part by an NSF grant DMS-9800790 and by an Alfred P. Sloan Research Fellowship. D. Waldram was supported in part at Princeton University by the DOE under contract No. DE-FG0291ER40671 and also wishes to acknowledge the hospitality of the Aspen Center for Physics where part of this work was done. 


\section{References}

[1] P. Horava and E. Witten, "Heterotic and Type I String Dynamics from Eleven Dimensions," Nucl. Phys. B460, 506 (1996) [hep-th/9510209].

[2] P. Horava and E. Witten, "Eleven-Dimensional Supergravity on a Manifold with Boundary," Nucl. Phys. B475, 94 (1996) [hep-th/9603142].

[3] E. Witten, "Strong Coupling Expansion Of Calabi-Yau Compactification," Nucl. Phys. B471, 135 (1996) [hep-th/9602070].

[4] T. Banks and M. Dine, "Couplings and Scales in Strongly Coupled Heterotic String Theory," Nucl. Phys. B479, 173 (1996) [hep-th/9605136]; I. Antoniadis and M. Quiros, "Large radii and string unification," Phys. Lett. B392, 61 (1997) [hep-th/9609209];

K. Benakli, "Phenomenology of low quantum gravity scale models," Phys. Rev. D60, 104002 (1999) [hep-ph/9809582].

[5] N. Arkani-Hamed, S. Dimopoulos and G. Dvali, "The hierarchy problem and new dimensions at a millimeter," Phys. Lett. B429, 263 (1998) [hep-ph/9803315];

I. Antoniadis, N. Arkani-Hamed, S. Dimopoulos and G. Dvali, "New dimensions at a millimeter to a Fermi and superstrings at a TeV," Phys. Lett. B436, 257 (1998) [hep-ph/9804398].

[6] L. Randall and R. Sundrum, "A large mass hierarchy from a small extra dimension," Phys. Rev. Lett. 83, 3370 (1999) [hep-ph/9905221];

L. Randall and R. Sundrum, "An alternative to compactification," Phys. Rev. Lett. 83, 4690 (1999) hep-th/9906064.

[7] A. Lukas, B. A. Ovrut and D. Waldram, "Non-standard embedding and five-branes in heterotic M-theory," Phys. Rev. D59, 106005 (1999) [hep-th/9808101].

[8] Z. Lalak, S. Pokorski and S. Thomas, "Beyond the standard embedding in M-theory on S(1)/Z(2)," Nucl. Phys. B549, 63 (1999) [hep$\mathrm{ph} / 9807503]$.

[9] S. Stieberger, " $(0,2)$ heterotic gauge couplings and their M-theory origin," Nucl. Phys. B541, 109 (1999) [hep-th/9807124].

[10] A. Lukas, B. A. Ovrut, K. S. Stelle and D. Waldram, "The universe as a domain wall," Phys. Rev. D59, 086001 (1999) [hep-th/9803235].

[11] A. Lukas, B. A. Ovrut, K. S. Stelle and D. Waldram, "Heterotic M-theory in five dimensions," Nucl. Phys. B552, 246 (1999) [hepth/9806051]. 
[12] S. Donaldson, Proc. London Math. Soc. 31 (1985).

[13] K. Uhlenbeck and S.-T. Yau, Comm. Pure App. Math. 39257 (1986), 42703 (1986).

[14] J. Distler and B. Greene, "Aspects Of $(2,0)$ String Compactifications," Nucl. Phys. B304, 1 (1988).

[15] S. Kachru, "Some three generation $(0,2)$ Calabi-Yau models," Phys. Lett. B349, 76 (1995) [hep-th/9501131].

[16] R. Friedman, J. Morgan and E. Witten, "Vector bundles and F theory," Commun. Math. Phys. 187, 679 (1997) [hep-th/9701162].

[17] R. Donagi, Asian. J. Math. 1, 214 (1997).

[18] M. Bershadsky, A. Johansen, T. Pantev and V. Sadov, "On fourdimensional compactifications of F-theory," Nucl. Phys. B505, 165 (1997) [hep-th/9701165].

[19] R. Donagi, A. Lukas, B. A. Ovrut and D. Waldram, "Non-perturbative vacua and particle physics in M-theory," JHEP 9905, 018 (1999) [hepth/9811168].

[20] R. Donagi, A. Lukas, B. A. Ovrut and D. Waldram, "Holomorphic vector bundles and non-perturbative vacua in M-theory," JHEP 9906, 034 (1999) [hep-th/9901009].

[21] G. Curio, "Chiral matter and transitions in heterotic string models," Phys. Lett. B435, 39 (1998) [hep-th/9803224].

[22] B. Andreas, "On vector bundles and chiral matter in $\mathrm{N}=1$ heterotic compactifications," JHEP 9901, 011 (1999) [hep-th/9802202].

[23] R. Donagi, B. A. Ovrut and D. Waldram, "Moduli spaces of fivebranes on elliptic Calabi-Yau threefolds," JHEP 9911, 030 (1999) [hepth/9904054].

[24] J. D. Breit, B. A. Ovrut and G. C. Segre, "E(6) Symmetry Breaking In The Superstring Theory," Phys. Lett. B158, 33 (1985);

[25] E. Witten, "Symmetry Breaking Patterns In Superstring Models," Nucl. Phys. B258, 75 (1985).

[26] R. Donagi, B. A. Ovrut, T. Pantev and D. Waldram, in preparation.

[27] B. Andreas, G. Curio and A. Klemm, "Towards the standard model spectrum from elliptic Calabi-Yau," hep-th/9903052. 
[28] A. Grassi, Internat. J. Math. 4, 203 (1993);

D. R. Morrison and C. Vafa, "Compactifications of F-Theory on CalabiYau Threefolds - II," Nucl. Phys. B476, 437 (1996) [hep-th/9603161].

[29] R. P. Thomas, "Examples of bundles on Calabi-Yau 3-folds for string theory compactifications," [math.AG/9912179].

[30] M. Li, "Boundary States of D-Branes and Dy-Strings," Nucl. Phys. B460, 351 (1996) [hep-th/9510161];

M. B. Green, J. A. Harvey and G. Moore, "I-brane inflow and anomalous couplings on D-branes," Class. Quant. Grav. 14, 47 (1997) [hepth/9605033].

[31] P. S. Aspinwall and R. Y. Donagi, "The heterotic string, the tangent bundle, and derived categories," Adv. Theor. Math. Phys. 2, 1041 (1998) [hep-th/9806094].

[32] P. Berglund and P. Mayr, "Heterotic string/F-theory duality from mirror symmetry," Adv. Theor. Math. Phys. 2, 1307 (1999) [hepth/9811217].

P. Berglund and P. Mayr, "Stability of vector bundles from F-theory," JHEP 9912, 009 (1999) [hep-th/9904114].

[33] G. Rajesh, "Toric geometry and F-theory/heterotic duality in four dimensions," JHEP 9812, 018 (1998) [hep-th/9811240]. 